Article

\title{
Morphology of Thin Film Composite Membranes Explored by Small-Angle Neutron Scattering and Positron-Annihilation Lifetime Spectroscopy
}

\author{
Vitaliy Pipich ${ }^{1}$, Marcel Dickmann ${ }^{2}$, Henrich Frielinghaus ${ }^{1}$ (D), Roni Kasher ${ }^{3}$, \\ Christoph Hugenschmidt ${ }^{2}$, Winfried Petry ${ }^{2}$, Yoram Oren ${ }^{3}$ and Dietmar Schwahn ${ }^{2, *(1)}$ \\ 1 Jülich Centre for Neutron Science JCNS-FRM II; Outstation at FRM II, Lichtenbergstr. 1, D-85747 Garching, \\ Germany; v.pipich@fz-juelich.de (V.P.); h.frielinghaus@fz-juelich.de (H.F.) \\ 2 Heinz Maier-Leibnitz Zentrum (MLZ), Technische Universität München, Lichtenbergstr. 1, D-85748 \\ Garching, Germany; marcel.dickmann@frm2.tum.de (M.D.); christoph.hugenschmidt@frm2.tum.de (C.H.); \\ winfried.petry@frm2.tum.de (W.P.) \\ 3 Zuckerberg Institute for Water Research, Jacob Blaustein Institutes for Desert Research, Ben-Gurion \\ University of the Negev, Sede Boqer Campus 84990, Israel; kasher@bgu.ac.il (R.K.); yoramo@bgu.ac.il (Y.O.) \\ * Correspondence: d.schwahn@fz-juelich.de
}

Received: 2 March 2020; Accepted: 16 March 2020; Published: 18 March 2020

check for updates

\begin{abstract}
The morphology of thin film composite (TFC) membranes used in reverse osmosis (RO) and nanofiltration (NF) water treatment was explored with small-angle neutron scattering (SANS) and positron-annihilation lifetime spectroscopy (PALS). The combination of both methods allowed the characterization of the bulk porous structure from a few $\AA$ to $\mu \mathrm{m}$ in radius. PALS shows pores of $\sim 4.5 \AA$ average radius in a surface layer of about $4 \mu \mathrm{m}$ thickness, which become $\sim 40 \%$ smaller at the free surface of the membranes. This observation may correlate with the glass state of the involved polymer. Pores of similar size appear in SANS as closely packed pores of $\sim 6 \AA$ radius distributed with an average distance of $\sim 30 \AA$. The main effort of SANS was the characterization of the morphology of the porous polysulfone support layer as well as the fibers of the nonwoven fabric layer. Contrast variation using the media $\mathrm{H}_{2} \mathrm{O} / \mathrm{D}_{2} \mathrm{O}$ and supercritical $\mathrm{CO}_{2}$ and $\mathrm{CD}_{4}$ identified the polymers of the support layers as well as internal heterogeneities.
\end{abstract}

Keywords: detection of the order of $\AA$ to micrometer large pores in RO membranes; fibers of nonwoven fabric support layer; chemistry and internal structures; positron-annihilation lifetime spectroscopy; small-angle neutron scattering using contrast variation

\section{Introduction}

Reverse osmosis (RO) and nanofiltration (NF) play a crucial role in the production of potable water from waste, brackish and seawater [1-3]. The main active components of RO desalination plants are thin film composite (TFC) membranes composed of three polymeric layers with a total thickness of $\sim 140$ to $\sim 300 \mu \mathrm{m}$. Figure 1 shows a schematic description of TFC-RO/NF membranes. The layer facing the treated water, i.e., the feed is a polyamide (PA) film of thickness in the range 0.1 to $0.3 \mu \mathrm{m}$ depending on membrane type deposited on top of a polyethersulfone (PES) or polysulfone (PSU) porous support layer of $\sim 40 \mu \mathrm{m}$ thickness. A second support layer is a nonwoven fabric made from polyester (PET) or polypropylene. 


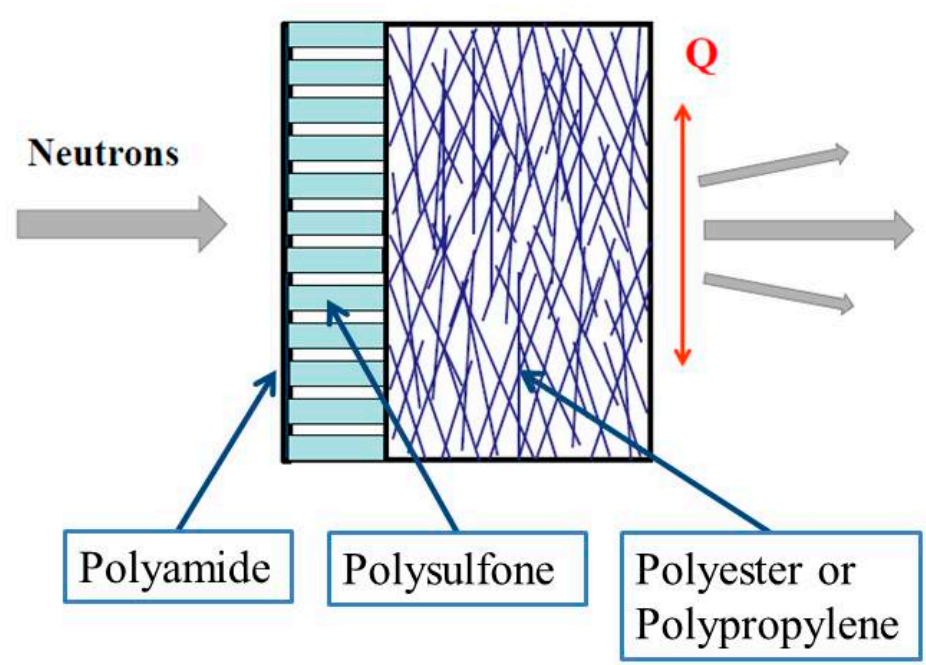

Figure 1. Design of thin film composite (TFC), reverse osmosis (RO), and nanofiltration (NF) membranes and pathway of the neutron beam through the membrane. The dimension of the membrane morphology such as pores is determined in direction of the scattering vector $(\mathbf{Q})$. Thickness of the polyamide skin layer is in the range 0.1 to $0.3 \mu \mathrm{m}$, the porous and nonwoven fabric support layers about $40 \mu \mathrm{m}$ and 100 to $300 \mu \mathrm{m}$, respectively.

In this paper, we analyze the morphology of several commercial RO and NF membranes from the perspectives of positron-annihilation lifetime spectroscopy (PALS) and small-angle neutron scattering (SANS). Both methods are non-invasive techniques, which usually do not require special sample treatment. PALS measures pores of a few $\AA$ radius in the membrane surface layer over a depth of about $3 \mu \mathrm{m}$ thickness thereby exploring the attendance of micro pores in the whole polyamide skin layer as well as in the outer part of the polysulfone support layer. Thus, PALS delivers relevant structural information of the PA selective layer, which determines water permeability and salt rejection. Neutrons, on the other hand, penetrate the whole membrane, thus characterizing pores and the fibers of the nonwoven fabric of radii between $\AA$ and several $\mu \mathrm{m}$ as well as identifying the polymers of the entire membrane.

However, the analysis of asymmetric TFC-RO/NF membranes with SANS is complicated and work-intensive as the scattering is strong and locally not specified. These difficulties were largely resolved by corrections for multiple scattering and by performing contrast variation measurements, which allow for identification and a more detailed morphological characterization of the membrane polymers. Scattering from the PA skin layer of the membrane is almost non-detectable with SANS due to its small thickness in comparison with both supporting layers. Only at large scattering angles analogous to large $\mathbf{Q}$ the morphology of the PA layer might become visible when scattering from pores of several Å size are dominating. PALS supports the existence of such pores as well as a combined study of SANS and PALS on a standalone PA skin layer in Ref. [4].

An important motivation of our effort is to demonstrate the strength of a joint exploitation of SANS and PALS for revealing the bulk morphology of RO and NF membranes. This effort provides a basis of interpretation for our operando RO-SANS desalination experiments including membrane compaction, membrane scaling, and biofouling as well as concentration polarization in the presence of organic molecules in a simulated secondary effluent (SSE) solution [5-7] or aqueous silica solution. Some of the very first results of those operando RO-SANS experiments are reported in [8]. 


\section{Materials and Methods}

\subsection{Thin Film Composite Membranes}

Six commercial TFC membranes with different nonwoven support layers are listed in Table 1. The producer of the RO98 pHt membrane claims a sodium chloride rejection of $\geq 98 \%$ and a tolerance of high $\mathrm{pH}$ and temperature. The Dow Filmtec RO membranes SW30HR, BW30LE, and XLE are, respectively, optimized for high rejection seawater, low energy brackish water, and extra low energy brackish water $\mathrm{RO}$ desalination. These membranes are composed of, respectively, polyamide, polysulfone, and polyester of $0.2 \mu \mathrm{m}, 40 \mu \mathrm{m}$, and $120 \mu \mathrm{m}$ thickness. The producer recommends the NF270 membrane for removing organic carbon (TOC) and trihalomethanes (THM) from surface and ground water while maintaining high salt passage and partial hardness removal. The TM820 seawater desalination membrane consists of fully cross-linked aromatic polyamide composites.

Table 1. Explored RO- and NF-TFC membranes.

\begin{tabular}{|c|c|c|c|c|c|}
\hline Producer & Membrane & Type & Composition & $\begin{array}{c}\text { Membrane } \\
\text { Thickness } \\
{[\mu \mathrm{m}]}\end{array}$ & $\begin{array}{l}\text { Experimental } \\
\text { Technique }\end{array}$ \\
\hline $\begin{array}{c}\text { Alfa } \\
\text { Laval }^{1}\end{array}$ & RO98 pHt & RO membrane & $\begin{array}{l}\text { polyamide polysulfone } \\
\text {-polypropylene }\end{array}$ & 300 & SANS/PALS \\
\hline \multirow{4}{*}{$\begin{array}{l}\text { Dow }^{2} \\
\text { Filmtec }\end{array}$} & SW30HR & $\begin{array}{l}\text { seawater high } \\
\text { rejection }\end{array}$ & \multirow{5}{*}{$\begin{array}{c}\text { polyamide-polysulfone } \\
\text {-polyester }\end{array}$} & 140 & SANS/PALS \\
\hline & BW30LE & $\begin{array}{l}\text { brackish water } \\
\text { low energy }\end{array}$ & & 150 & SANS/PALS \\
\hline & XLE [8] & $\begin{array}{l}\text { low pressure } \\
\text { RO membrane }\end{array}$ & & \multirow{3}{*}{140} & SANS \\
\hline & NF270 & $\begin{array}{l}\text { nanofiltration } \\
\text { membrane (NF) }\end{array}$ & & & SANS/PALS \\
\hline $\begin{array}{l}\text { Toray }^{3} \\
\text { (Japan) }\end{array}$ & TM820 & $\begin{array}{l}\text { sea water RO } \\
\text { membrane }\end{array}$ & & & SANS \\
\hline
\end{tabular}

${ }^{1}$ Thanks to Stephan Wild (Coordinator Environment) and Dipl. W.- Ing. Micha Kruse (Sales Engineer Service) from Alfa Laval Mid Europe GmbH in DE-21509 Glinde (Germany) for providing the RO membrane RO98 $\mathrm{pHt} ;{ }^{2} \mathrm{The}$ RO membranes SW30HR, BW30LE, XLE, and NF270 were provided from DOW FILMTEC (Minneapolis, MN, USA); ${ }^{3}$ Seawater RO membranes TM820 were supplied from Toray Industries in Tokio (Japan).

The XLE membrane was grafted with methacrylic acid (MA) by redox-initiated graft polymerization in order to decrease biofouling propensity as previously described [9]. Grafting of RO membranes with MA is an example of surface modification for reducing fouling propensity. This strategy is based on surface hydrophilization of the membrane, thereby acquiring low adherence of organic substances and biological molecules, resulting in lower organic- and biofouling of polymeric membranes in water treatment processes [10,11]. The grafting of XLE membranes with MA was carried out by immersing the membranes in an aqueous MA solution followed by adding potassium metabisulfite $\left(\mathrm{K}_{2} \mathrm{~S}_{2} \mathrm{O}_{5}\right)$ and potassium persulfate $\left(\mathrm{K}_{2} \mathrm{~S}_{2} \mathrm{O}_{8}\right)$ [9]. The solution with the membrane was agitated gently on Unimax 1010 orbital shaker (Heidolph, Kelheim, Germany) for $20 \mathrm{~min}$. The reaction was stopped by discarding the solution and washing the membrane rigorously with water.

The TM820 membrane was immersed for 1-5 days in a solution that simulated desalination of treated domestic wastewater at a stage of $80 \%$ recovery [5]. Then, the membranes were gently rinsed with 50 vol \% ethanol/water, and dried. 


\subsection{Small-Angle Neutron Scattering (SANS) Instruments}

The neutron experiments were performed at two SANS instruments (KWS 1 and KWS 3), both operating at the MLZ, Garching, Germany [12], covering a $\mathbf{Q}$ from $10^{-4}$ to $0.2 \AA^{-1}$, thereby allowing the detection of pores of $\AA$ to $\mu \mathrm{m}$ in radius. Most experiments were performed at KWS3, the instrument for very small scattering angles (VSANS) and only part of the experiments was performed at the classical pin-hole instrument, KWS 1 (SANS), covering a range between $10^{-3}$ to $0.4 \AA^{-1}$. The modulus of $Q$ is determined according to $Q=4 \pi / \lambda \sin (\delta / 2)$ from the scattering angle $(\delta)$ and the neutron wavelength $(\lambda)$. The scattering vector is proportional to the change of neutron momentum $\mathbf{p}=\hbar \mathbf{Q}$ (de Broglie relationship) and its modulus indicates the range where objects of $1 / \mathrm{Q}$ dimension mainly contribute to scattering.

Experiments detecting neutrons scattered at very small angles (KWS 3) became possible by implementing a high-quality elliptical mirror focusing the neutrons onto the detector mirroring a few $\mathrm{mm}^{2}$ large area of the aperture at the entrance of the instrument with only negligible contributions from outside this area $[13,14]$. The detector was $9.50 \mathrm{~m}$ from the sample position and $\lambda=12.8 \AA$ with a spread in wavelength of $\Delta \lambda / \lambda=20 \%$. The experiments at KWS 1 were performed with $\lambda=7 \AA(\Delta \lambda / \lambda=10 \%)$ and a variable sample to detector distance between 20 to $1.5 \mathrm{~m}$. Appendices A.1-A.3 summarize the necessary scattering laws for the analysis of the scattering patterns.

As already mentioned, the scattering pattern of neutrons comprises the morphological information of the entire membrane, which makes it challenging to distinguish between the contributions of the individual layers. This difficulty can be resolved when exposing the same membrane with a liquid contrast medium, whose solvent coherent scattering length density $\left(\rho_{\mathrm{S}}\right)$ can easily be changed. A standard contrast medium is the mixture of $\mathrm{H}_{2} \mathrm{O}$ and $\mathrm{D}_{2} \mathrm{O}$ of varying composition, and lesser known examples are supercritical fluids (SCFs) such as $\mathrm{CO}_{2}$ and/or $\mathrm{CD}_{4}$ at variable pressure; we explain these in detail in Appendix A.3.

Table 2 presents parameters of the membrane polymers such as coherent scattering length density $(\rho)$ and incoherent scattering cross-section $\left(\mathrm{d} \Sigma / \mathrm{d} \Omega_{\text {inc. }}\right)$ needed for analyzing the scattering data. As discussed in the theoretical section of Appendices A.1 and A.2 as well as in [15], the scattering length density $(\rho)$ determines the strength of scattering. It is defined as the sum of the neutron coherent scattering length of the atoms $\left(b_{j}\right)$ forming the molecule, divided by the molecular volume $\left(\Omega_{M}\right)$, that is $\rho=\sum_{j} b_{j} / \Omega_{M}$. The incoherent scattering cross-section $\left(d \Sigma / d \Omega_{\text {inc. }}\right)$ describes the inherently non-coherent part of the neutron scattering, is of equal strength in all directions and is considered as (uninteresting) background. All pore radii errors calculated from SANS data reflect only the statistical error of the fitting routine. 
Table 2. Relevant parameters of membrane material such as glass transition temperature $\left(\mathrm{T}_{\mathrm{G}}\right)$, incoherent scattering cross-section $\left(\mathrm{d} \Sigma / \mathrm{d} \Omega_{\text {inc }}\right)$, coherent scattering length density $(\rho), \mathrm{D}_{2} \mathrm{O}$ volume fraction $\left(\Phi_{\text {match }}\right)$ of water gaining the same $\rho$ of the membrane polymer, i.e., to make it invisible for neutrons.

\begin{tabular}{|c|c|c|c|c|c|c|c|}
\hline Molecule & $\begin{array}{l}\text { Chemical } \\
\text { Formula }\end{array}$ & $\begin{array}{l}\text { Mass Density } \\
{\left[\mathrm{g} / \mathrm{cm}^{3}\right]}\end{array}$ & $\begin{array}{l}\text { Molar Weight } \\
{[\mathrm{g} / \mathrm{mol}]}\end{array}$ & $\begin{array}{l}\text { Glass Transition } \\
\text { Temp. } \mathrm{T}_{\mathrm{G}}\left[{ }^{\circ} \mathrm{C}\right]\end{array}$ & $\begin{array}{c}\text { Scattering } \\
\text { Cross-Section } \\
\mathrm{d} \Sigma / \mathrm{d} \Omega_{\text {inc. }}\left[\mathrm{cm}^{-1}\right]\end{array}$ & $\begin{array}{c}\text { Scattering Length } \\
\text { Density } \rho[1010 \\
\left.\mathrm{cm}^{-2}\right]\end{array}$ & $\begin{array}{l}\Phi_{\text {match }} \\
\left(\mathrm{D}_{2} \mathrm{O}\right)\end{array}$ \\
\hline $\begin{array}{l}\text { Aromatic Polyamide (PA) } \\
\text { (Kevlar) }\end{array}$ & $\mathrm{N}_{2} \mathrm{C}_{14} \mathrm{H}_{10} \mathrm{O}_{2}$ & 1.44 & 238 & $60-75$ & 0.233 & $3.10 \pm 0.07$ & 0.53 \\
\hline Polysulfone (PSU) & $\mathrm{C}_{27} \mathrm{H}_{22} \mathrm{O}_{4} \mathrm{~S}$ & 1.24 & 442.54 & \multirow{2}{*}{$\sim 220$} & 0.237 & 2.08 & 0.38 \\
\hline Polyethersulfone (PES) & $\mathrm{C}_{12} \mathrm{H}_{10} \mathrm{O}_{4} \mathrm{~S}$ & $1.3-1.4$ & 250.27 & & 0.225 & 2.22 & 0.40 \\
\hline Polypropylene & $\mathrm{C}_{3} \mathrm{H}_{6}$ & 0.91 & 42.08 & -10 & 0.50 & -0.325 & 0.034 \\
\hline $\begin{array}{c}\text { Polyethylenterephthalate (PET, } \\
\text { Polyester) }\end{array}$ & \multirow{3}{*}{$\mathrm{C}_{10} \mathrm{H}_{8} \mathrm{O}_{4}$} & $1.38[16,17]$ & \multirow{3}{*}{192.17} & \multirow{3}{*}{$\sim 79$} & 0.37 & 2.58 & 0.45 \\
\hline PET (amorphous) & & $1.33[16]$ & & & 0.36 & 2.49 & 0.44 \\
\hline PET (crystalline) & & $1.52[17]$ & & & 0.41 & 2.84 & 0.49 \\
\hline Low density Polyethylene (PE) & $\mathrm{C}_{2} \mathrm{H}_{4}$ & 0.88 & 28.05 & -125 & 0.48 & -0.31 & 0.036 \\
\hline Methacrylic acid (MA) [18] & $\mathrm{C}_{4} \mathrm{H}_{6} \mathrm{O}_{2}$ & 1.015 & $84.83 ;(\mathrm{R}=3.23 \AA)$ & - & 0.27 & 1.12 & 0.24 \\
\hline
\end{tabular}




\subsection{Positron-Annihilation Lifetime Spectroscopy (PALS)}

Positron-annihilation lifetime spectroscopy (PALS) is a well-established technique for the analysis of the free volume in polymers (e.g., [19]). In polymers, the lifetime of ortho-Positronium (o-Ps), a bound state of positron and electron, is correlated with the pore size that o-Ps annihilate by interacting with surrounding electrons (pick-off process). The relation between the lifetime $(\tau)$ of o-Ps and the pore radius is described in the Tao-Eldrup model (see Appendix A.4 and Equation (A11)). Within this model the pore is approximated by a sphere with an outer radius $R_{0}$, and an inner radius $R$ with a zone $\Delta \mathrm{R}=\mathrm{R}_{0}-\mathrm{R}$ in which the electron density is not zero.

A pulsed mono-energetic positron beam of variable energy allows a depth resolved determination of the pore size of amorphous matter. The positron implantation profile in the material characterized by the mean implantation depth can be chosen by varying the positron energy [20] of the pulsed low energy positron system (PLEPS) [21,22] operated in vacuum at the neutron-induced positron source at the MLZ (NEPOMUC) [23]. The mean implantation depth $(\bar{z})$ for a given positron energy $(\mathrm{E})$ can be approximated by Equation (A12).

Positron lifetime spectra containing more than $4 \times 10^{6}$ counts were recorded as functions of implantation energy. The resolution function was obtained measuring a p-doped silicon carbide ( $p$-SiC) reference sample with a well-known positron lifetime. This was followed by numerical deconvolution of the recorded spectra and fitted with a least-squares method based on the Levenberg-Marquardt algorithm. The fit residuals and the chi-square $\left(\chi^{2}\right)$ value show reasonable results, if three lifetimes $\left(\tau_{1}, \tau_{2}, \tau_{3}\right)$ were considered in the fit routine. The lifetimes $\tau_{1}$ and $\tau_{2}$, which are in the 100 ps range, are related to the direct positron annihilation and decay of para-Ps, whereas the longest component $\tau_{3}$ is associated with the above-mentioned pick-off lifetime of o-Ps in polymeric matter. Reasonable fits were only obtained for several spectra if considering an additional longer lifetime component with $\tau_{4}$ $>\tau_{3}$ - see Appendix A.4.

The statistical error bars for the lifetimes are small-typical in the order of 1-2\% due to good counting statistics. Systematic errors are larger due to assumptions such as spherical shape of the pores or the uncertainty in the definition of pore radius seen by positrons. In consequence, we did not provide error bars for the absolute radii from PALS.

\section{Results and Discussion}

A first impression about the characteristics of scattering was gained for the Alfa Laval (RO98 $\mathrm{pHt}$ ) membrane in Figure 2a. These data were measured at KWS3 and KWS1 together covering a Q interval from $10^{-4}$ to $0.2 \AA^{-1}$ and are corrected for multiple scattering as outlined in Appendix A.2. Similar scattering data over the same $Q$ range were already presented for the XLE membrane in Figure 7 of a previous article [8]. The main differences between the Alfa Laval and Dow Filmtec membranes are, respectively, the overall thickness of $300 \mu \mathrm{m}$ and $140 \mu \mathrm{m}$ as well as the nonwoven fabric layers made from polypropylene and polyester fibers. Three groups of scattering entities of the order of $1.6 \mu \mathrm{m}, 0.34 \mu \mathrm{m}$, and $12 \AA$ for the radius of gyration $\left(\mathrm{R}_{\mathrm{g}}\right)$ become visible from the characteristic shape of the scattering pattern, which later will be attributed to the layers of the RO membrane. It should be kept in mind that scattering in the Q range below $\sim 10^{-2} \AA^{-1}$ is dominated by the two supporting layers. Scattering from the active skin layer could become visible beyond $5 \times 10^{-2} \AA^{-1}$ from scattering centers of about $12 \AA$ radius, which was fitted with the form factor of spheres (Equation (A3)) in a $Q$ range from 0.04 to $0.3 \AA^{-1}$ considering the upturn $\left(\mathrm{Q}^{-4}\right.$ Porod law) of scattering from the pores of the support layer.

The main focus of the present section is the identification and analysis of the two $\mu \mathrm{m}$ large scattering centers achieved from SANS contrast variation and from fitting the corresponding scattering laws. The subsequent section will discuss order of $\AA$ large pores observed with PALS mainly in the polyamide surface layer and with SANS at large $Q$.

Before getting to that point, we demonstrate the effect of multiple scattering in Figure $2 \mathrm{~b}$ comparing the as measured with the on basis of the Equation (A7) corrected scattering pattern of the $\mathrm{RO} 98 \mathrm{pHt}$ 
membrane exposed to $40 \mathrm{vol} \% \mathrm{D}_{2} \mathrm{O}$ aqueous mixture. Despite the membrane thickness of only $300 \mu \mathrm{m}$, multiple scattering becomes considerable because of the large scattering contrast of the medium with respect to the polypropylene fibers (Table 2). We corrected all SANS data measured at low $Q$ for multiple scattering.

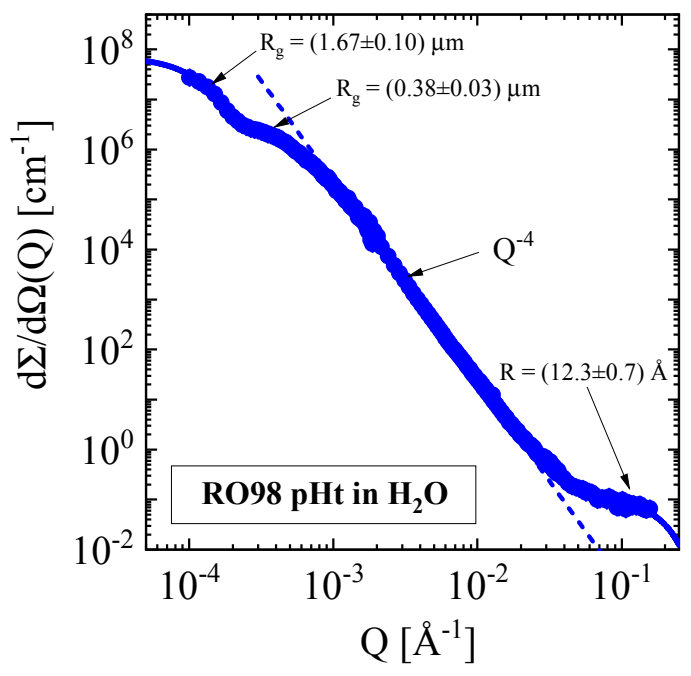

(a)

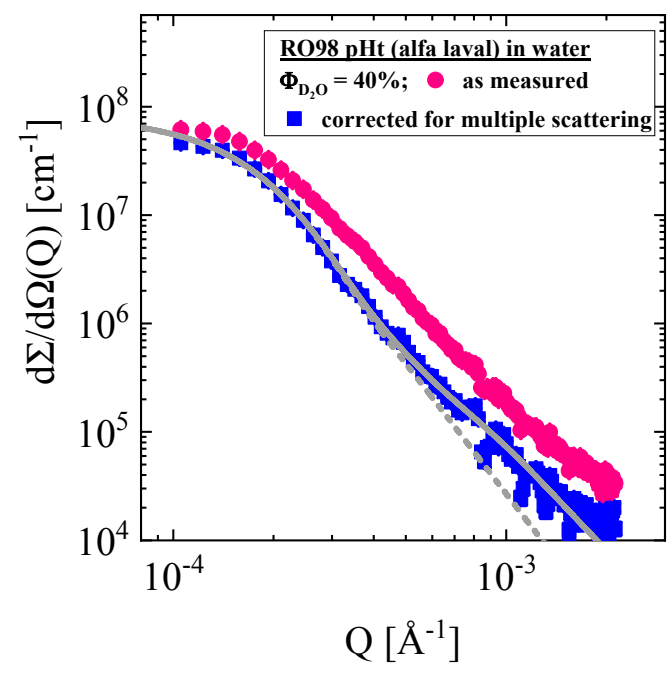

(b)

Figure 2. (a) Scattering pattern of the $\mathrm{RO} 98 \mathrm{pHt}$ membrane exposed to $\mathrm{H}_{2} \mathrm{O}$ after correction for multiple scattering. (b) Visualization of the effect of multiple scattering at small Q. Solid and dashed lines represent a fit of the scattering law (Equation (A4)). Contrast medium is water composed of $40 \mathrm{vol} \% \mathrm{D}_{2} \mathrm{O}$.

\subsection{Identification of the Scattering Centers from SANS Contrast Variation}

\subsubsection{RO98 pHt Membrane with Polypropylene Nonwoven Support}

Contrast variation experiments of the $\mathrm{RO}$ membranes were performed in the lower $\mathrm{Q}$ regime of the VSANS instrument showing enhanced scattering of $\mu \mathrm{m}$ large scattering centers. Scattering curves of the Alfa Laval membrane are depicted in Figure 3a,b for various scattering contrasts affected by the mixture of $\mathrm{H}_{2} \mathrm{O} / \mathrm{D}_{2} \mathrm{O}$ and the SCF$-\mathrm{CO}_{2}$ at $38^{\circ} \mathrm{C}$. Figures $\mathrm{A} 1$ and $\mathrm{A} 2 \mathrm{a}$ show the corresponding scattering length densities $\left(\rho_{\mathrm{S}}\right)$ of both media. Comparing the scattering data in Figure 3 allows us to observe the advantage of SCFs as contrast media where much finer tuning of $\rho_{S}$ is achieved for the same membrane and only one membrane sample is required. On the other hand, when $\mathrm{D}_{2} \mathrm{O}-\mathrm{H}_{2} \mathrm{O}$ mixtures are used as the contrast medium, an individual piece of membrane is required for each composition in order to make sure that the membrane is in equilibrium with the correct $\mathrm{D}_{2} \mathrm{O}-\mathrm{H}_{2} \mathrm{O}$ composition. The main disadvantage of SCF is its smaller contrast range.

The two scattering centers of larger and smaller size already observed in Figure 2a show an individual behavior, namely an increasing and declining intensity with increasing scattering contrast. Both scattering centers were fitted with Equation (A4) whose superposed scattering intensity, i.e., $\mathrm{d} \Sigma / \mathrm{d} \Omega(\mathrm{Q})=\sum_{\mathrm{i}=1,2} \mathrm{~d} \Sigma / \mathrm{d} \Omega_{\mathrm{i}}(\mathrm{Q})$, is depicted as solid lines showing excellent agreement with the experimental data. The fit parameters of the two classes of scattering centers are plotted in Figure 4 versus $\rho_{S}$ of the contrast medium and are compiled for all membranes in Tables 3 and 4 . The size of the scattering centers, i.e., their $\mathrm{R}_{g}$, is depicted in Figure $4 \mathrm{a}$, b versus $\rho_{S}$ of the contrast media $\mathrm{H}_{2} \mathrm{O} / \mathrm{D}_{2} \mathrm{O}$ and $\mathrm{SCF}-\mathrm{CO}_{2}$. The parameters in Figure $4 \mathrm{c}, \mathrm{d}$ are proportional to the scattered intensity at $\mathrm{Q}=0$ and thereby to the scattering contrast $\Delta \rho^{2}=\left(\rho_{P, i}-\rho_{S}\right)^{2}$ of the membrane polymer $\left(\rho_{P, i}\right)$ and the contrast medium. The smallest intensity in case of $\rho_{m}=\rho_{S}$ (see vertical line) refers to the type of polymer via comparison of $\rho_{\mathrm{m}}$ with the calculated $\rho$ in Table 2 . The polymers polysulfone and polypropylene are clearly identified as substances for the porous support and nonwoven fabric layers, respectively. The two parameters $d \Sigma / d \Omega_{i=1}(Q=0)$ and $P_{\alpha, i=2}(\alpha=3)$ in Equation (A4) were chosen for identification 
in the large $(\mathrm{i}=1)$ and small $(\mathrm{i}=2)$ particles, respectively, because both parameters are minimally influenced by each other in their corresponding $Q$ range. $d \Sigma / d \Omega_{i=1}(Q=0)$ and the amplitude $P_{\alpha, i=2}(\alpha$ $=3$ ) dominate, respectively, at small and large $Q$ by orders of magnitude.

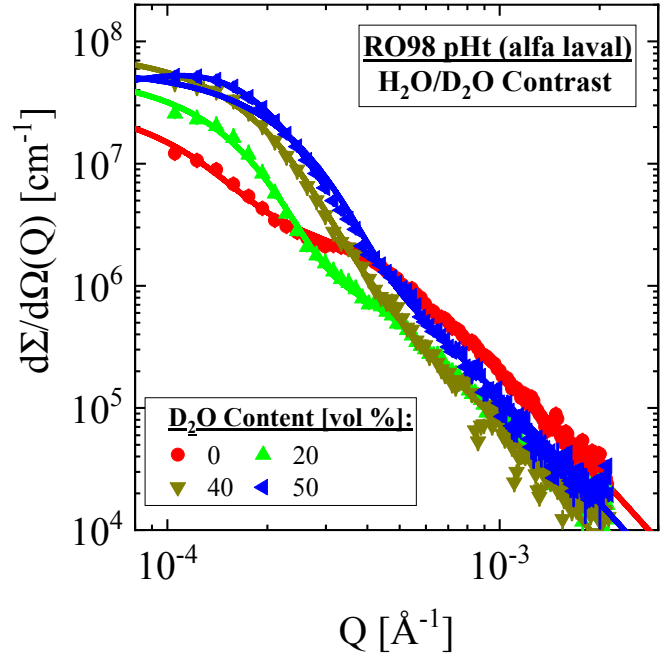

(a)

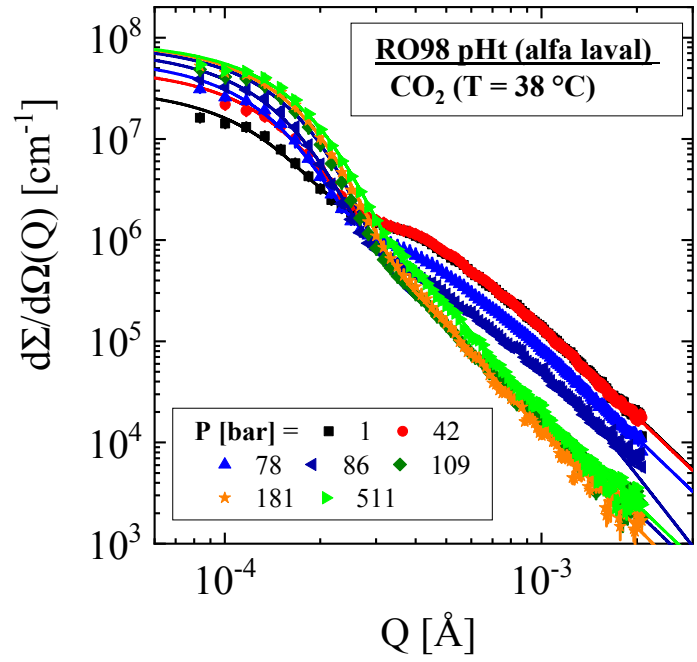

(b)

Figure 3. Scattering patterns of the $\mathrm{RO} 98 \mathrm{pHt}$ membrane at varying scattering contrast of the contrast medium (a) $\mathrm{H}_{2} \mathrm{O} / \mathrm{D}_{2} \mathrm{O}$ and (b) $\mathrm{SCF}-\mathrm{CO}_{2}$. Both contrast media show enhanced and reduced intensity with increasing $\mathrm{D}_{2} \mathrm{O}$ and $\mathrm{CO}_{2}$ concentration at small and large $\mathrm{Q}$, respectively. The scattering curves were fitted with Equation (A4) shown as solid lines. All data are corrected for multiple scattering as outlined in Figure 2.

An interesting observation of the nonwoven fabric layer is the continuous decline of $\mathrm{R}_{\mathrm{g}}$ with enhanced $\rho_{\mathrm{S}}$, whereas $R_{\mathrm{g}}$ (pore size) of the polysulfone layer stays constant with $\mathrm{H}_{2} \mathrm{O} / \mathrm{D}_{2} \mathrm{O}$ and shows only a slight enhancement of up to about $25 \%$ when exposed to $\mathrm{SCF}-\mathrm{CO}_{2}$ at increasing pressures. The intensity parameter of the nonwoven material is plotted as $\sqrt{\mathrm{d} \Sigma / \mathrm{d} \Omega(0) / R_{\mathrm{g}}^{6}}$ versus $\rho_{\mathrm{S}}$ delivering a straight line, which becomes zero at negative $\rho_{\mathrm{m}}$. (upper part of Figure $4 \mathrm{c}, \mathrm{d}$ ). This combination of SANS parameters had to be chosen because of the large variation of $R_{g}$ and its dependence of $\mathrm{d} \Sigma / \mathrm{d} \Omega(0) \propto \mathrm{N}_{\mathrm{P}} \mathrm{V}_{\mathrm{P}}^{2} \propto \mathrm{N}_{\mathrm{P}} \mathrm{R}_{\mathrm{g}}^{6}\left(\Phi_{\mathrm{P}}=\mathrm{N}_{\mathrm{P}} \mathrm{V}_{\mathrm{P}}\right)$ (Equation (A2)). A constant pore number density $\left(\mathrm{N}_{\mathrm{P}}\right)$ was assumed. Matching at $\rho_{\mathrm{m}}$ occurs for the negative values of -1.11 and -0.47 in units of $10^{10}$ $\mathrm{cm}^{-2}$ in good agreement with the theoretical value for polypropylene $\left(-0.325 \times 10^{10} \mathrm{~cm}^{-2}\right.$; Table 2). The amplitudes of $P_{3}$ are 1.85 and $2.05 \times 10^{10} \mathrm{~cm}^{-2}$ at $\rho_{\mathrm{m}}$ corresponding to the evaluated $\rho=2.08 \times$ $10^{10} \mathrm{~cm}^{-2}$ of polysulfone in Table 2 (Lower part of Figure 4c,d). SANS contrast variation identified both polymers in compliance with the manufacturer's information.

The "intensity" parameter of the fibers of the nonwoven fabric layer shows two distinctively different slopes below and beyond $\rho_{S}=0.72 \times 10^{10} \mathrm{~cm}^{-2}$ (upper part of Figure 4d), which is identified as the $\mathrm{CO}_{2}$ gas/SCF phase boundary at $38{ }^{\circ} \mathrm{C}$ and 74 bar (Figure A2 and [24]). We ascribe this observation to the degree of wetting caused from capillary forces of the $\mathrm{CO}_{2}$ solvent and the fibers. The distinct slopes and amplitudes of the "intensity" parameter results from preferential wetting of the $\mathrm{CO}_{2}$ gas thereby reducing the scattering contrast $\left(\Delta \rho^{2}\right)$. These considerations are consistent with the lower amplitude of $\mathrm{P}_{3}$ in the $\mathrm{CO}_{2}$ gas phase in comparison with the extrapolated $\mathrm{P}_{3}$ from the SCF phase. The finite scattering of the polysulfone layer at the matching condition is another interesting observation. We interpret this observation from pores and/or some other heterogeneity inside the fiber the contrast medium is not able to fill. We will discuss this issue later in context with the other membranes, which opens a relevant access to the internal morphology of membranes not being involved in the filtering process. 


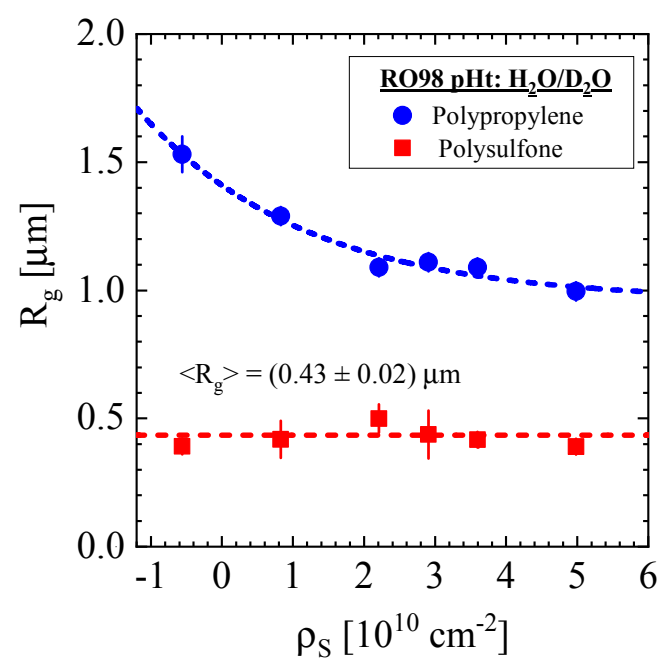

(a)

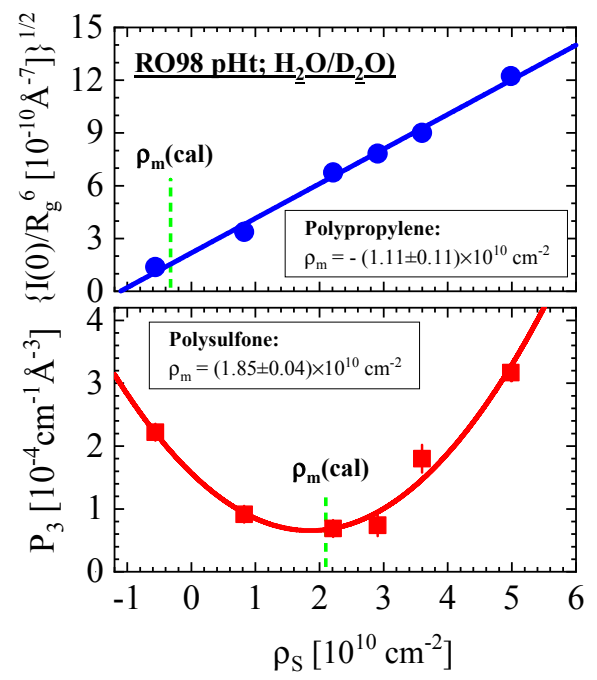

(c)

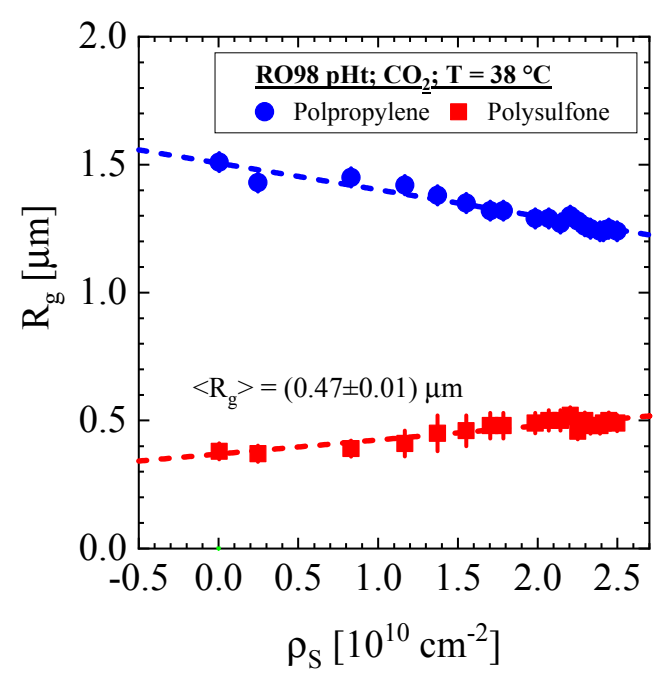

(b)

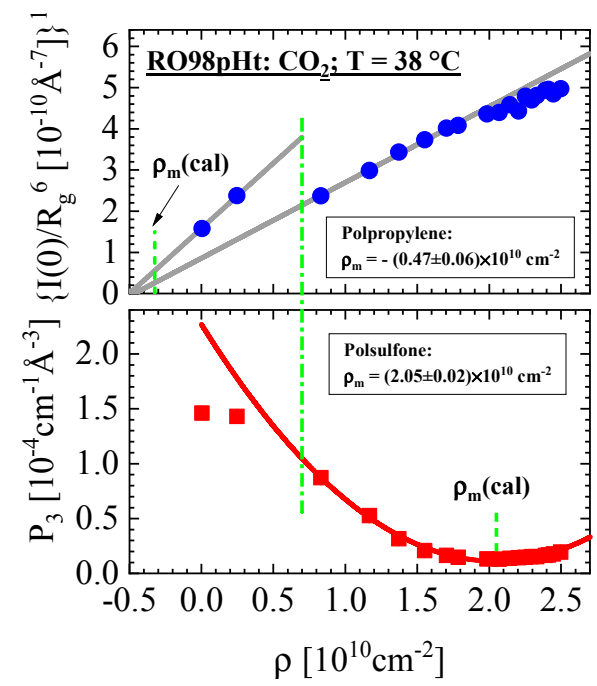

(d)

Figure 4. The small-angle neutron scattering (SANS) parameters of the RO98 $\mathrm{pHt}$ membrane are plotted versus $\rho_{\mathrm{S}}$ of the contrast media $(\mathbf{a}, \mathbf{c}) \mathrm{H}_{2} \mathrm{O} / \mathrm{D}_{2} \mathrm{O}$ and $(\mathbf{b}, \mathbf{d})$ supercritical fluid $(\mathrm{SCF})-\mathrm{CO}_{2}$. The large and small pores are attributed to the polypropylene nonwoven fabric and to the polysulfone porous support layer, respectively. (d) The dashed-dotted line represents the gas/SCF phase boundary of $\mathrm{CO}_{2}$ at $38^{\circ} \mathrm{C}$. The symbol I $(0)$ was chosen instead of $\mathrm{d} \Sigma / \mathrm{d} \Omega(0)$ because of space limitation.

\subsubsection{SW30HR Sea Water RO Membrane with Polyester Nonwoven Support Layer}

Figure 5 shows contrast variation experiments with the contrast media $\mathrm{SCF}-\mathrm{CO}_{2}$ at 10 and $38{ }^{\circ} \mathrm{C}$ and $\mathrm{SCF}-\mathrm{CD}_{4}$ at $10^{\circ} \mathrm{C}$ (see Figure A2b). Figure 5a shows two characteristic scattering patterns for the membrane in vacuum and exposed to $\mathrm{SCF}-\mathrm{CO}_{2}$ at $38^{\circ} \mathrm{C}$ and $\mathrm{P}=134$ bar corresponding to $\rho_{\mathrm{S}}$ $=0$ and $1.87 \times 10^{10} \mathrm{~cm}^{-2}$, respectively. The dashed curves represent fits of the individual scattering centers from both supporting layers. The larger $R_{g}$ increases with $\rho_{S}$ by about $25 \%$ whereas the smaller $R_{g}$ stays constant (Figure $5 b$ ). The "intensity" parameters in Figure $5 c$,d deliver values of $\rho_{\mathrm{m}}$ in consistence with the polyester polyethylenterephthalat (PET) and polyethersulfone (PES) for the nonwoven and porous support layers, respectively. $R_{g}$ of the nonwoven material in Figure $5 b$ shows a small hump at $\rho_{S}=(2.5 \pm 0.03) \times 10^{10} \mathrm{~cm}^{-2}$, which is in correspondence with the matching value $\rho_{\mathrm{m}}$ and is therefore identified as the radius of gyration $\left(\mathrm{R}_{\mathrm{g}, \mathrm{l}}\right)$ of "closed" heterogeneities not accessible for the contrast medium. We fitted the experimental $R_{g, 1}$ with the phenomenological equation $\mathrm{R}_{\mathrm{g}}\left(\rho_{\mathrm{S}}\right)=\mathrm{A}+\mathrm{B} \times\left(\rho_{\mathrm{m}}-\rho_{\mathrm{S}}\right)^{\beta}$ with $\beta=0.5, \mathrm{~A}=(1.85 \pm 0.02) \mu \mathrm{m}, \mathrm{B}=-(3.95 \pm 0.11) \times 10^{-6} \mu \mathrm{m}$ 
$\times \mathrm{cm}^{2 \beta}$ shown as a dashed line. On basis of Equation (A8) we obtain $R_{g, o p}=(1.07 \pm 0.03) \mu \mathrm{m}$ and $R_{g, c l}$ $=(1.82 \pm 0.04) \mu \mathrm{m}$ for the nonwoven fabric morphology achievable and not achievable for the contrast medium, respectively.

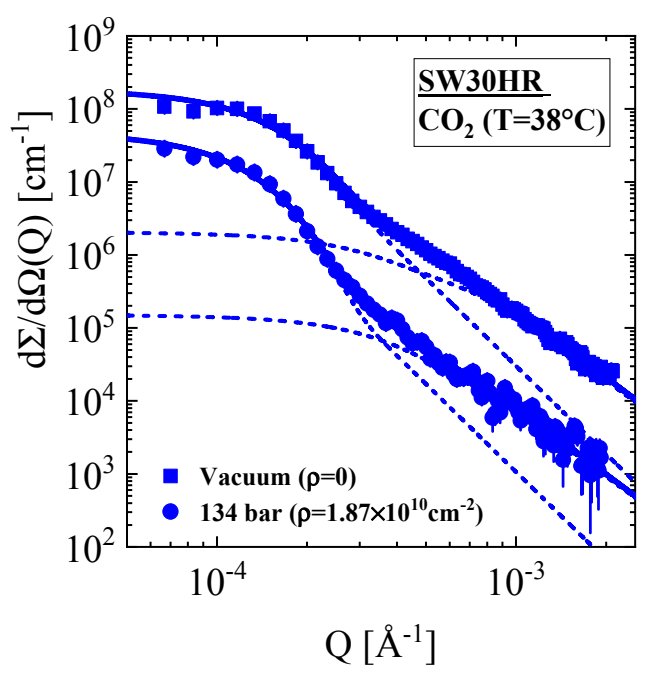

(a)

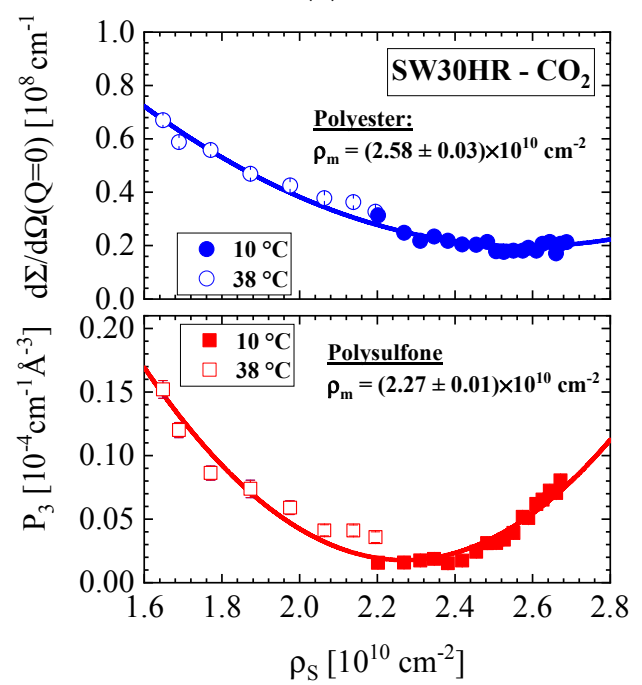

(c)

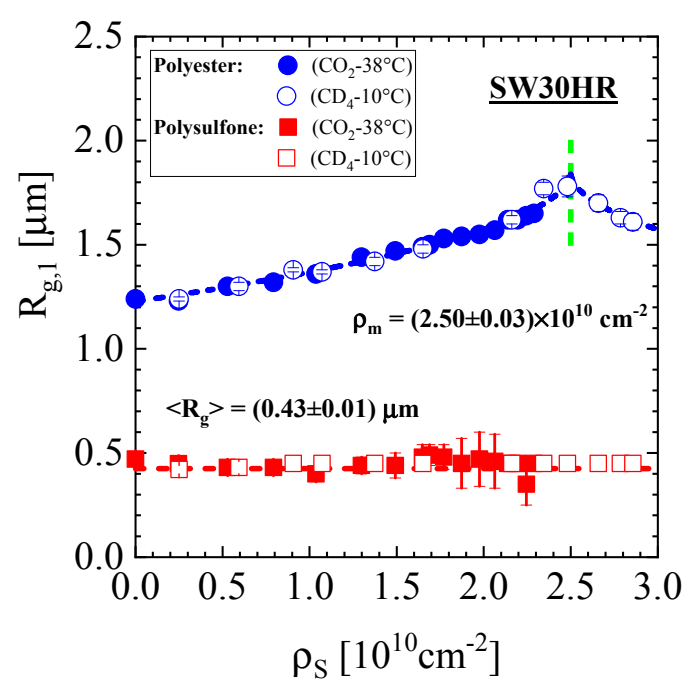

(b)

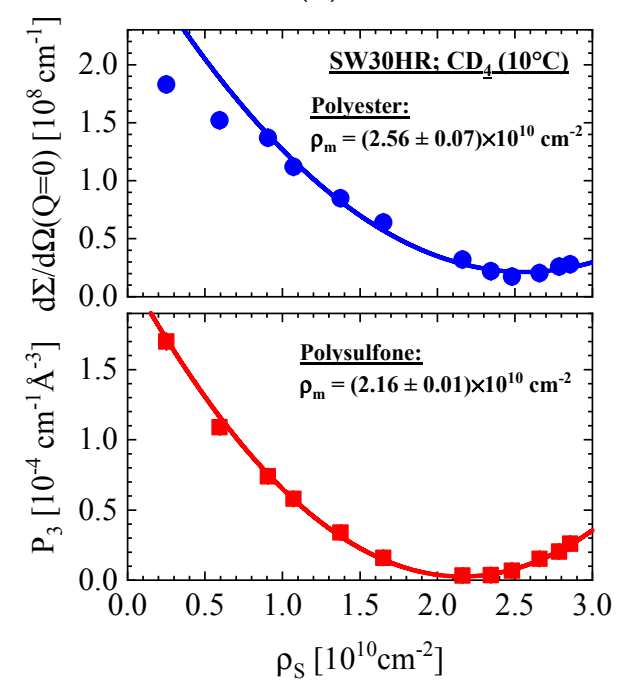

(d)

Figure 5. (a) Scattering from the SW30HR seawater RO membrane in vacuum and exposed to $\mathrm{SCF}-\mathrm{CO}_{2}$ at $38{ }^{\circ} \mathrm{C}$ and 134 bar; (b) $R_{g}$ of the polysulfone and polyester layer versus $\rho_{S}$. (c,d) $d \Sigma / d \Omega(0)$ of the polysulfone and polyester layer versus $\rho_{\mathrm{S}}$ with the contrast media: (c) $\mathrm{SCF}-\mathrm{CO}_{2}$ along the isothermal pathways at 10 and $38^{\circ} \mathrm{C}$ and $(\mathbf{d}) \mathrm{CD}_{4}$ at $10{ }^{\circ} \mathrm{C}$.

\subsubsection{BW30LE Low Energy Brackish Water RO Membrane}

Figure 6 shows the scattering patterns of the BW30LE membrane. The two scattering patterns in Figure 6a represent the membrane in vacuum and exposed to SCF-CD 4 at $10{ }^{\circ} \mathrm{C}$ and 244 bar, which causes a strong decline of scattering. The dashed curves represent the SW30HR membrane for the individual scattering centers of both layers. Figure $6 b$ depicts the $R_{g}$ 's versus $\rho_{S}$. The larger $R_{g}$ shows a similar variation as the SW30HR membrane with a peak at $\rho_{\mathrm{m}}=(2.59 \pm 0.02) \times 10^{10} \mathrm{~cm}^{-2}$ but for the polysulfone layer a factor of about two smaller $\mathrm{R}_{\mathrm{g}}$. The "intensity" parameters (Figure 6c) deliver $\rho_{\mathrm{m}}$ values consistent with $\rho$ evaluated for the polyester PET and PES of the nonwoven and porous support layers, respectively. Again, $R_{g}$ shows a weak hump at the matching condition of the nonwoven fabric layer. We achieve $R_{g, o p}=(1.17 \pm 0.02) \mu \mathrm{m}$ and $R_{g, c l}=(1.82 \pm 0.02) \mu \mathrm{m}$ from a fit with 
$\beta=0.5$ applying the same procedure as in the last section. It should be mentioned that the pore size of the polysulfone support layer is about $40 \%$ smaller (averaged value: $R_{g}=(0.27 \pm 0.01) \mu \mathrm{m}$ ) than that for the SW30HR membrane.

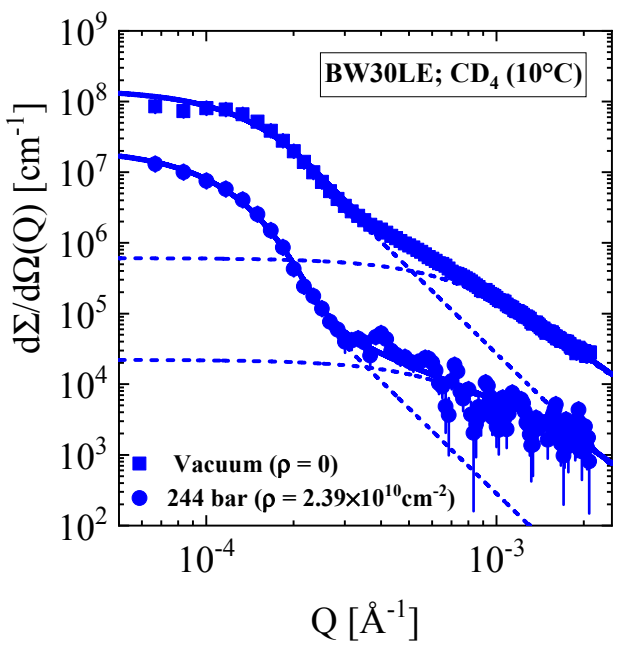

(a)

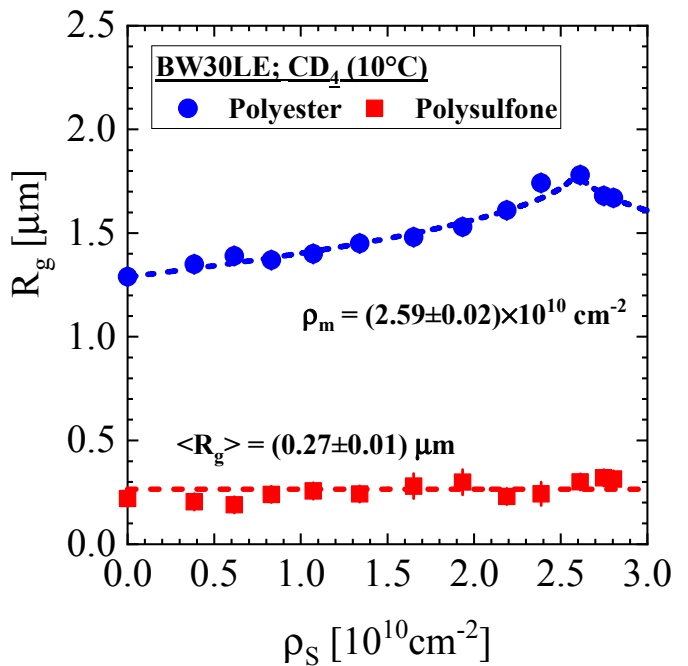

(b)

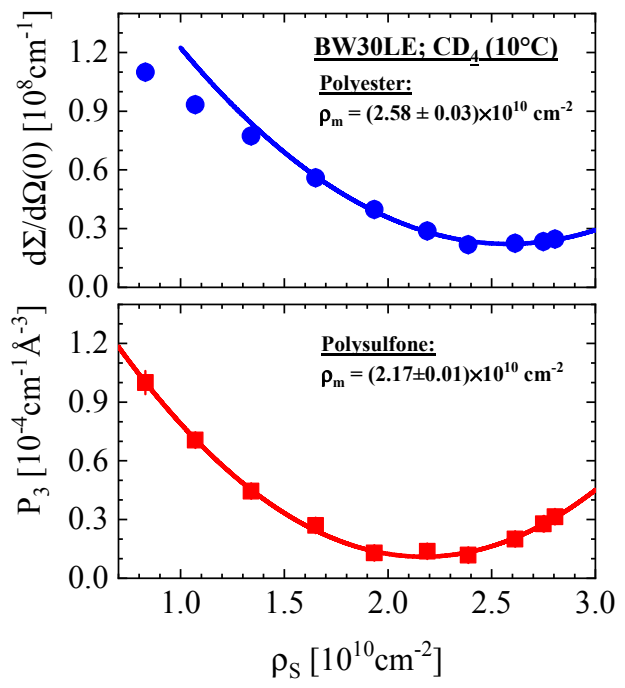

(c)

Figure 6. (a) Scattering pattern of the BW30LE RO membrane in vacuum and exposed to $\mathrm{CD}_{4}$ at 10 ${ }^{\circ} \mathrm{C}$ and $\mathrm{P}=244$ bar. (b) $\mathrm{R}_{\mathrm{g}}$ of the nonwoven and porous layer versus $\rho_{\mathrm{S}}$ (c) The scattering intensity at $\mathrm{Q}=0$ of the polysulfone and polyester layer versus $\rho_{\mathrm{S}}$.

\subsubsection{Pristine and Grafted XLE BWRO Membrane}

Figure 7 shows the SANS results of a pristine and MA grafted XLE membrane. The contrast medium was $\mathrm{H}_{2} \mathrm{O} / \mathrm{D}_{2} \mathrm{O}$. Figure 7 a shows two scattering patterns of the pristine membrane exposed to $\mathrm{H}_{2} \mathrm{O}$ and to an aqueous mixture of $40 \mathrm{vol} \% \mathrm{D}_{2} \mathrm{O}$ showing the change of the larger and smaller scattering centers with the contrast medium. The dashed lines represent the fits of the smaller scattering centers. Figure $7 \mathrm{~b}$ depicts the intensity dependent parameters plotted versus $\rho_{S}$ similarly as for the other membranes. We find differences for the nonwoven material with respect to the other RO membranes: A constant (i.e., independent from $\left.\rho_{\mathrm{S}}\right)$ and smaller $\mathrm{R}_{\mathrm{g}}=(0.91 \pm 0.04) \mu \mathrm{m}$ as well as a smaller $\rho\left(\rho_{\mathrm{m}}=\right.$ $\left.(1.80 \pm 0.08) \times 10^{10} \mathrm{~cm}^{-2}\right)$ were determined, suggesting a different fiber material. The pristine porous support layer shows a constant $\mathrm{R}_{\mathrm{g}}$ of $(0.23 \pm 0.02) \mu \mathrm{m}$ and a matching value of $\rho_{\mathrm{m}}$ consistent with polysulfone. The porous support layer is considerably influenced by MA grafting, showing a slightly 
larger $R_{g}$ of $(0.28 \pm 0.01) \mu \mathrm{m}$, a $20 \%$ larger $\rho_{\mathrm{m}}$ and an appreciably larger intensity at $\rho_{\mathrm{m}}$. The graft polymerization of the $\mathrm{RO}$ membrane occurs on the active skin layer as well as inside the porous support. Hence, poly methacrylate partially fills the pores of the PES layer, thereby showing a smaller volume fraction of pores achievable for water. The larger $R_{g}$ might result from the averaging process (z-average) of the pore size distribution, i.e., stronger weighting of the larger pores. MA grafting seemingly prefers filling the smaller pores in the bulk of the porous support layer.

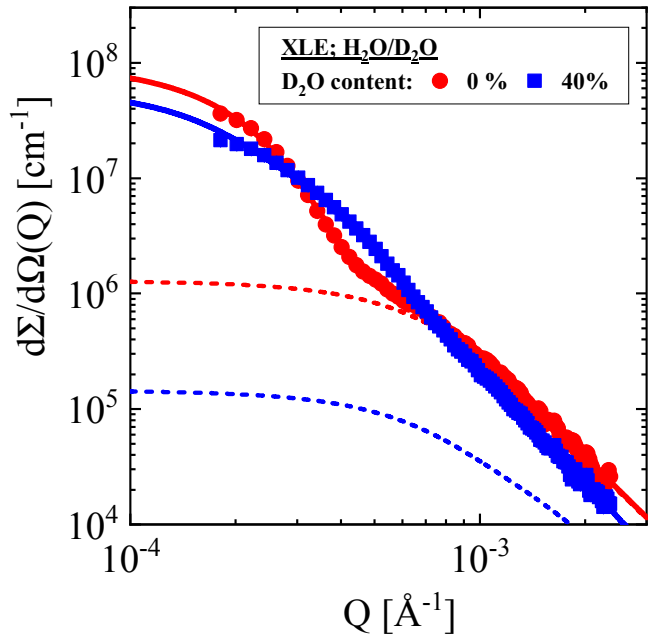

(a)

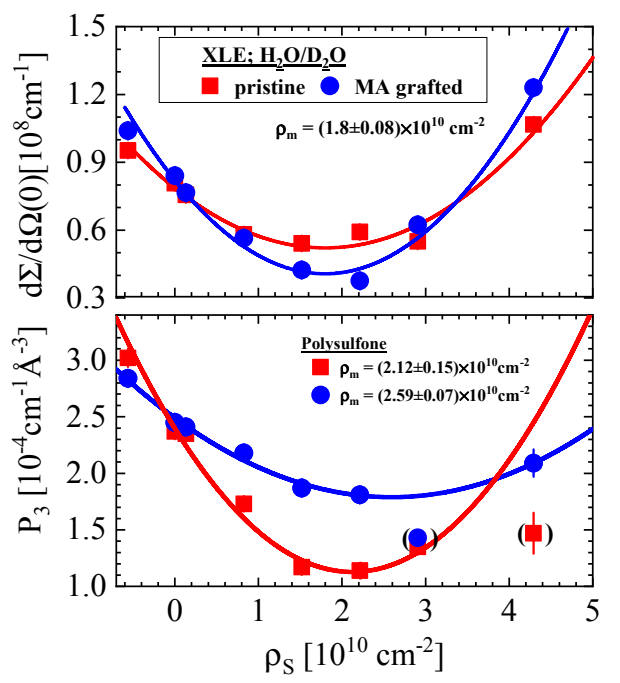

(b)

Figure 7. (a) SANS patterns of the pristine XLE membrane in $\mathrm{H}_{2} \mathrm{O}$ and in a mixture of $\mathrm{H}_{2} \mathrm{O} / \mathrm{D}_{2} \mathrm{O}$ with $40 \mathrm{vol} \% \mathrm{D}_{2} \mathrm{O}$ [8]. (b) Intensity parameters of pristine and grafted XLE versus $\rho_{\mathrm{S}}$. The upper and lower parts show the parameter of the nonwoven and polysulfone layer of the membrane, respectively.

\subsection{Discussion of the SANS Data from the Supporting Layers}

The relevant SANS parameters of the nonwoven fabric and porous support layers are compiled below in Tables 3 and 4. As already mentioned, scattering from the polyamide surface layer is too weak to be detected in the $Q$ range of less than $10^{-3} \AA^{-1}$ (Figures 3-7 ) due to its thickness of less than one $\mu \mathrm{m}$ in comparison with the support layers. We, however, determined the morphology of 10 standalone polyamide layers of $\mu \mathrm{m}$ thickness with SANS and PALS as reported in [4].

\subsubsection{Nonwoven Fabric Support}

The SANS parameters of the nonwoven fabric layer are compiled in Table 3. The scattering length density at matching condition ( $\rho_{\mathrm{m}}$ in the fourth column) gives information about the polymer of the fibers, i.e., it identifies the polymer by comparing it with the evaluated $\rho$ in Table 2 . According to the producer's information, the nonwoven fabric of the RO98 $\mathrm{pHt}$ membrane is made from polypropylene, showing a theoretical value of -0.325 (all values in units of $10^{10} \mathrm{~cm}^{-2}$, Table 2). The experimental $\rho_{\mathrm{m}}$ determined from SCF- $\mathrm{CO}_{2}$ contrast variation is -0.47 , in good agreement with the calculated one, whereas the contrast medium water is -1.11 , a worse result. A linear fit of the "water" data in the upper Figure 4c excluding the smallest parameter measured in $\mathrm{H}_{2} \mathrm{O}$ (smallest $\rho_{S}$ ) displays a $\rho_{\mathrm{m}}$ of $(0.88 \pm 0.15)$, which is still too large. We speculate that $\mathrm{SCF}-\mathrm{CO}_{2}$ has better wetting condition as water. The fibers of the nonwoven fabric layers of the SW30HR and BW30LE membranes were identified as polyethylenterephthalat (PET) in excellent agreement with its theoretical $\rho=2.58 \times 10^{10}$ $\mathrm{cm}^{-2}$. On the other hand, the corresponding $\rho_{\mathrm{m}}$ of the XLE nonwoven fabric delivers a value of $1.80 \times 10^{10} \mathrm{~cm}^{-2}$, which is too small to be identified as pure PET. Bi-component fibers composed, e.g., of 27 vol \% low-density polyethylene (PE) and 73 vol \% PET (see Table 3), would fit the SANS data. The remarkably improved tensile strength of such bi-component fibers is sometimes the preferred material for nonwoven fabric [25]. 
Table 3. SANS parameters of the nonwoven fabric normalized to the thickness of the membrane. The parameter $\Phi_{\text {int }}$ represents the volume fraction of heterogeneities of the fibers as determined from the ratio of scattering at $\rho=0$ and $\rho_{\mathrm{m}}$. (see Figure $5 \mathrm{c}, \mathrm{d}$, Figure $6 \mathrm{c}$, or Figure $7 \mathrm{~b}$ ). The parameters of the RO98 pHt membrane were determined at sufficiently strong scattering contrast realized for the contrast media of water of $40 \mathrm{vol} \% \mathrm{D}_{2} \mathrm{O}$ and SC- $\mathrm{CO}_{2}$ at 181 bar.

\begin{tabular}{|c|c|c|c|c|c|c|c|c|c|}
\hline Membrane & Polymer & Medium & $\begin{array}{c}\rho \mathrm{m}[1010 \\
\left.\mathrm{cm}^{-2}\right]\end{array}$ & $\begin{array}{c}\mathrm{d} \Sigma / \mathrm{d} \Omega(0)[107 \\
\left.\mathrm{cm}^{-1}\right]\end{array}$ & $\operatorname{Rg}[\mu \mathrm{m}]$ & $\begin{array}{c}\text { Ratio } \gamma \\
\text { (Equation (A8)) }\end{array}$ & $\begin{array}{l}\mathrm{Q} 2[10-4 \\
\left.\mathrm{cm}^{-1} \AA^{-3}\right]\end{array}$ & $\begin{array}{c}\text { Фfil [vol } \\
\% \text { ] }\end{array}$ & $\begin{array}{c}\text { Sint [vol } \\
\text { \%] }\end{array}$ \\
\hline \multirow[t]{2}{*}{$\mathrm{RO} 98 \mathrm{pHt}$} & PP & $\begin{array}{c}40 \mathrm{vol} \% \mathrm{D} 2 \mathrm{O}(\Delta \rho= \\
\left.2.68 \times 1010 \mathrm{~cm}^{-2}\right)\end{array}$ & $-(1.11 \pm 0.11)$ & $8.27 \pm 0.22$ & $1.10 \pm 0.01$ & - & 2.35 & 1.66 & - \\
\hline & $\mathrm{PP}$ & $\begin{array}{c}\mathrm{CO} 238^{\circ} \mathrm{C}, 181 \mathrm{bars} \\
\left(\Delta \rho=2.07 \times 1010 \mathrm{~cm}^{-2}\right)\end{array}$ & $-(0.47 \pm 0.06)$ & $9.14 \pm 0.2$ & $1.30 \pm 0.01$ & - & 1.09 & 1.29 & - \\
\hline SW30HR & PET & \multirow{4}{*}{ Vacuum } & $2.59 \pm 0.03$ & $18.1 \pm 0.6$ & $1.25 \pm 0.01$ & 0.12 & 3.2 & 2.9 & 0.4 \\
\hline BW30LE & PET & & $2.58 \pm 0.03$ & $14.6 \pm 0.5$ & $1.26 \pm 0.02$ & 0.15 & 2.64 & 1.7 & 0.3 \\
\hline XLE & PET0.73/PE0.27 & & $1.80 \pm 0.08$ & $8.08 \pm 0.05$ & $0.91 \pm 0.04$ & 0.65 & 2.89 & 1.6 & 2.9 \\
\hline Grafted XLE & & & $1.79 \pm 0.08$ & $8.40 \pm 0.06$ & $0.94 \pm 0.01$ & 0.48 & 2.18 & 1.8 & 1.6 \\
\hline
\end{tabular}


The scattering from the nonwoven fabric layers originates from centers of the order of $\mu \mathrm{m}$ in radius, i.e., $\mathrm{R}_{\mathrm{g}}$ and volume fraction of $\sim 2 \%$ (column 9 and 10). Polymer fibers are the minor phase of the nonwoven fabric material loosely packed with a large free space of $\sim 98 \%$ in accordance with the Babinet principle [26] (p. 32). Figure 8a depicts a typical conformation and interplay of fibers of a polyester nonwoven fabric, schematically amplified in Figure $8 \mathrm{~b}$. The coherent scattering characterizes rods of length $\xi$ and cross-section $R$, whose $R_{g}$ is expressed as representing a solid rod of length $\xi$ and cross-section radius $R$ [26] (p. 159). The length $\xi$ represents the average distance of the fibers between two nodes an interpretation, which has much in common with the blob model for semi-dilute polymer solutions [27] (Chapter III).

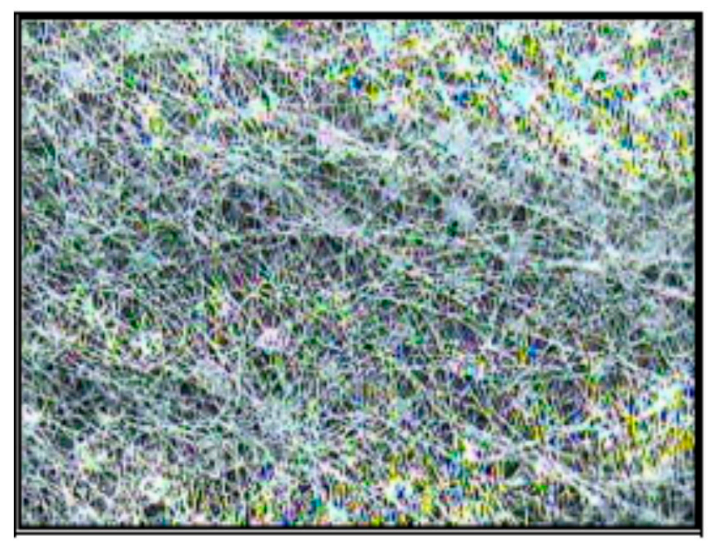

(a)

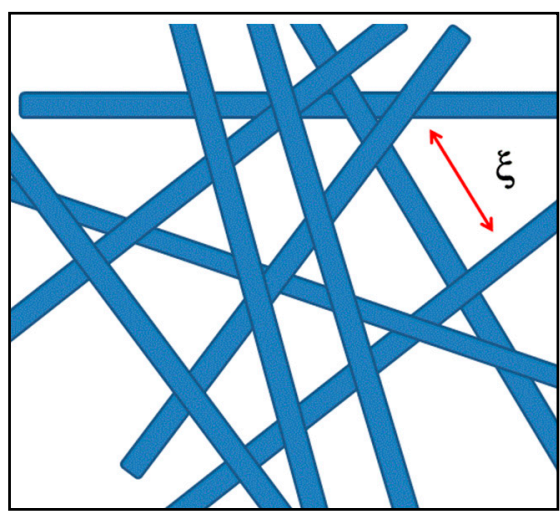

(b)

Figure 8. (a) Microscope view (40x) of nonwoven fabric of $12 \mathrm{~g} / \mathrm{m}^{2}$ polyester (taken from p. 22 of ref. [25]) and (b) sketch of a fiber network with the average length $\xi$ of the segment between two nodes of the network.

We observe two types of scattering centers, one affected by the contrast medium the other one not affected by the contrast medium. The first one represents the global fiber, whereas the second one internal heterogeneities of the fiber such as closed pores. Internal heterogeneities of fibers could be of different origin such as phases of crystalline and amorphous regions, closed pores, or binary phases as expected of bi-component fibers proposed for the XLE membranes. The ratio of scattering intensity from internal heterogeneities with respect to the total fiber is expressed as $\gamma$ in the 7th column of Table 3 as evaluated from $d \Sigma / d \Omega(0)$ and $P_{3}$ in Figure 4, Figure 5, Figure 6, Figure 7 for the nonwoven fabric and porous support layers, respectively at $\rho=\rho_{\mathrm{m}}$ and $\rho=0$.

The volume fraction of the fibers and internal structure listed in the 9 th and 10th column of Table 3 were derived from the second moment (Q2) of the intensity (Equation (A6)), the scattering contrast at $\rho_{\mathrm{m}}$, and multiplied with $(1-\gamma)$ and $\gamma$ (for definition of $\gamma$, see Equation (A10)), respectively. The integrals of Q2 were determined from the fitted scattering pattern of the polysulfone and nonwoven support layer as, for instance, shown as a dashed line in Figures 5a and 6a. The choice of the fitted curve allows integration over larger $Q$. These parameters, however, represent values averaged over the total volume of the membrane due to absolute calibration of the scattered intensity. For the Dow Filmtec membranes, the volume fraction of the fibers $\Phi_{\text {fil }}$ has to be multiplied with 140/100 assuming a porous support layer of $40 \mu \mathrm{m}$ thickness. Considering this correction, we find a fiber volume fraction between $2 \%$ and $4 \%$ which corresponds to $0.028-0.056 \mathrm{~g} / \mathrm{cm}^{3}$ mass density of PET nonwoven fabric in fair agreement with values from literature of $0.019-0.035 \mathrm{~g} / \mathrm{cm}^{3}$ for nonwoven fabric of polyester fibers in [28] (Table 2). In addition, the volume fraction $\Phi_{\text {int }}$ has to be divided by $\Phi_{\text {fil }}$ delivering for the SW and BW membranes a volume fraction between $17 \%$ and $21 \%$ if representing closed pores $\left(\Phi_{\mathrm{cl}}\right)$. 
Internal fiber heterogeneities could be of different origin. Crystalline and amorphous regions are always present in fibers due to the production process for improved mechanical strength as extensively studied with SANS [29-31]. The scattering contrast of crystalline/amorphous phases of PET fibers is with $\Delta \rho=0.13 \times 10^{10} \mathrm{~cm}^{-2}$ negligibly weak; a $50 \%$ volume fraction of crystalline-amorphous morphology would deliver a Q2 $=8.3 \times 10^{-6} \mathrm{~cm}^{-1} \AA^{-3}$ which accounts for about $1 \%$ of the measured one. Therefore, we interpret the internal scattering of the fibers of the SW and BW membranes as from internal pores. In contrast, the determined large $\gamma$ of the XLE membrane, i.e., their strong internal scattering, is consistent with the proposed bi-component composition of the fibers with the PET and PE polymers. The scattering contrast of PET and PE has been evaluated from the corresponding $\rho$ in Table 2 as $\Delta \rho=2.89 \times 10^{10} \mathrm{~cm}^{-2}$ delivering a Q2 $=4.12 \times 10^{-3} \mathrm{~cm}^{-1} \AA^{-3}$ for a $50 \mathrm{vol} \%$ PET and PE melt (Equation (A6)). After multiplication, this Q2 with the averaged fiber volume fraction of $1.7 \%$ gains 7.0 $\times 10^{-5} \mathrm{~cm}^{-1} \AA^{-3}$, in consistence with the experimental example of $\mathrm{Q} 2=7.3 \times 10^{-5} \mathrm{~cm}^{-1} \AA^{-3}$, which was obtained after multiplication Q2 $=2.89 \times 10^{-4} \mathrm{~cm}^{-1} \AA^{-3}$ with $\gamma=0.65$ (Table 3) and normalization to the correct scattering contrast $\left(1.80 \times 10^{10} / 2.89 \times 10^{10}\right)^{2}$. $\mathrm{R}_{\mathrm{g}}$ of the XLE membrane is independent from $\rho_{S}$ in contrast to the SW30HR and BW30LE membranes discussed in context in Figures 5b and $6 \mathrm{~b}$.

\subsubsection{Porous Support Layer}

The SANS parameters of the porous support layer are compiled in Table 4. Except for the MA grafted XLE membrane, the scattering length density of the porous support layer of all membranes deliver an average value of $\rho_{\mathrm{m}}$ of $(2.13 \pm 0.05) \times 10^{10} \mathrm{~cm}^{-2}$. This value better corresponds to the PSU than PES polymer according to the listed theoretical $\rho$ in Table 2, confirming the producer's information.

The listed intensity ratio $\gamma$ is $44 \%$ larger for the RO98 $\mathrm{pHt}$ membrane when exposed to water at ambient pressure, whereas SCF- $\mathrm{CO}_{2}$ with $\gamma=5 \%$ fills the pores much better (95\%), to the same degree as the SW30HR and BW30LE membranes. On the other hand, both XLE membranes show a larger degree of internal structure, i.e., pores not achievable for the contrast media. The MA grafting causes a $\sim 20 \%$ enhanced $\rho_{\mathrm{m}}$ of $2.6 \times 10^{10} \mathrm{~cm}^{-2}$, whereas the pore radii $\left(R_{\mathrm{g}}\right)$ are constant within the given measurement error. The Dow Filmtec membranes show open pores with an average volume fraction of $4.2 \%$ when normalized to the total volume of the membranes. As these pores are part of the polysulfone support layer, the pore volume fraction has to be normalized to the thickness of the support layer $(40 \mu \mathrm{m})$, i.e., has to be multiplied by the ratio of membrane thickness $(140 \mu \mathrm{m})$ and support layer thickness $(40 \mu \mathrm{m})$, i.e., $140 / 40=3.5$, which delivers a pore volume fraction of nearly $15 \%$, consistent with results of ref. [32] (Table 1), reporting a surface porosity (area coverage) between $5.9 \%$ and $14.5 \%$. However, it should be stressed that SANS determines the volume fraction of pores in bulk. It should also be noted that the MA grafting is expected to partially fill the pores of the underlying support layer (see Figure 1, the PES side of the membrane) of the XLE membrane, since graft polymerization occurs both on the active skin layer and inside the porous support. 
Table 4. Parameters obtained from contrast variation of the porous support layer $\left(^{*}\right)$ measured in vacuum or $\mathrm{H}_{2} \mathrm{O}$ ). $\Phi \times 140 / 40=3.5$ or $300 / 40=7.5$.

\begin{tabular}{|c|c|c|c|c|c|c|c|c|c|}
\hline & Polymer & Medium & $\rho_{\mathrm{m}}\left[1010 \mathrm{~cm}^{-2}\right]$ & $\begin{array}{c}\mathrm{d} \Sigma / \mathrm{d} \Omega(0)[106 \\
\left.\mathrm{cm}^{-1}\right]\end{array}$ & $\mathbf{R}_{\mathrm{g}} *[\mu \mathrm{m}]$ & $\gamma$ & $\begin{array}{c}\mathrm{Q} 2\left[10^{-4}\right. \\
\left.\mathrm{cm}^{-1} \AA^{-3}\right]\end{array}$ & $\begin{array}{c}\Phi_{\text {pore }}[\text { vol } \\
\%]\end{array}$ & $\begin{array}{c}\Phi_{\text {int }}[\text { [vol } \\
\%]\end{array}$ \\
\hline \multirow[t]{2}{*}{ RO98 pHt } & PSU & $\begin{array}{c}\mathrm{H}_{2} \mathrm{O}(\Delta \rho= \\
\left.2.36 \times 10^{10} \mathrm{~cm}^{-2}\right)\end{array}$ & $1.85 \pm 0.04$ & $3.89 \pm 0.05$ & $0.43 \pm 0.02$ & 0.44 & 4.32 & 2.2 & 1.7 \\
\hline & & \multirow{5}{*}{ Vacuum } & $2.05 \pm 0.02$ & $2.27 \pm 0.01$ & $0.47 \pm 0.01$ & 0.05 & 4.01 & 4.6 & 0.2 \\
\hline SW30HR & PES & & $(2.16$ to 2.27$) \pm 0.01$ & $2.04 \pm 0.27$ & $0.43 \pm 0.01$ & 0.02 & 4.06 & 4.3 & 0.1 \\
\hline BW30LE & PES & & $2.17 \pm 0.01$ & $0.70 \pm 0.03$ & $0.22 \pm 0.02$ & 0.05 & 4.62 & 4.7 & 0.3 \\
\hline XLE & PSU & & $2.12 \pm 0.15$ & $1.08 \pm 0.02$ & $0.23 \pm 0.02$ & 0.48 & 6.25 & 3.6 & 3.4 \\
\hline MA grafted XLE & & & $2.59 \pm 0.07$ & $1.77 \pm 0.05$ & $0.28 \pm 0.01$ & 0.73 & 6.50 & 1.3 & 3.6 \\
\hline
\end{tabular}




\subsection{PALS Data}

The lifetime $\tau_{3}$ obtained by PALS estimates the mean size of pores on basis of the Tao-Eldrup model (Equation (A11)), which assumes o-Ps annihilation in spherically shaped pores of radius R [33]. The mean positron implantation depth is calculated according to Equation (A12) from the positron energy, assuming a mass density of $1.3 \mathrm{~g} / \mathrm{cm}^{3}$ for the samples (Table 2). PALS determines pores of nanometer size at the position of the implantation depth, at distances up to about $4 \mu \mathrm{m}$ from the membrane surface. This includes the polyamide skin layer as well as the adjacent part of the polysulfone layer. The intensity $\mathrm{I}_{3}$ is related to the total free volume in the polymer and allows observing changes of the free volume. The positron lifetime spectra were analyzed by minimizing the residuum between a fit comprising three to four lifetime components and the experimental data using the standard software PALSfit [34]. Figure A3 in the Appendix A shows a typical fit of a positron lifetime spectrum for the RO98 pHt membrane.

Figure 9a,b shows the measured intensity and lifetimes in the cleaned RO98 pHt membrane. The cleaning procedure of the $\mathrm{RO} 98 \mathrm{pHt}$ membrane was standard: First, exposure of the membrane to $50 \%$ water (DI) and isopropanol solution, then to pure water (DI) both for 24 hours before drying took place for five days in fresh air. An additional longer lifetime $\tau_{4}$ appears in the lifetime spectra with values between 60 and 90 ns. These large lifetimes are beyond the Tao-Eldrup model. However, a mean pore radius in the order of 40-90 ̊ can be estimated considering the o-Ps mean free path and measurements at room temperature [33,35], which is consistent with the observation of individual voids of about $75 \AA$ in the PA surface layer [36]. The near surface region $(<600 \AA)$ of the sample exhibits small free volume, which is indicated by the low intensity of both $\tau_{3}$, and $\tau_{4}$ (Figure $9 a$ ) and the decline of $\tau_{3}$. This is according to a smaller mean pore volume of around $60 \AA^{3}(R=2.5 \AA$ ) (Figure 10a) as will be discussed in Section 4 .

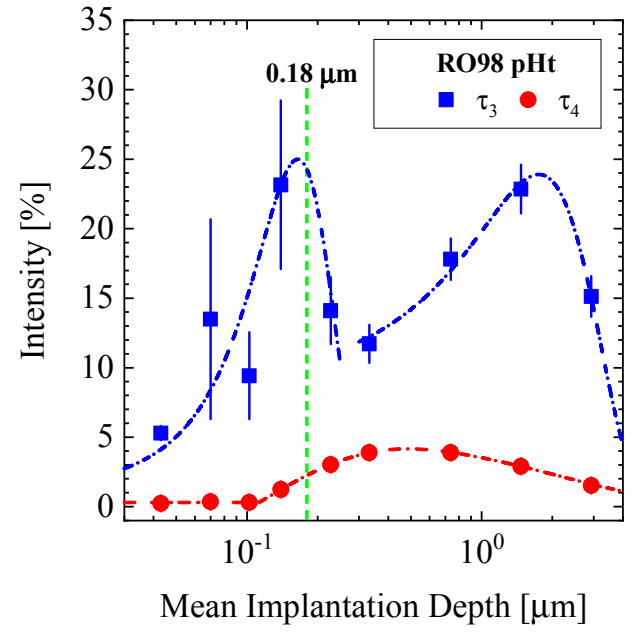

(a)

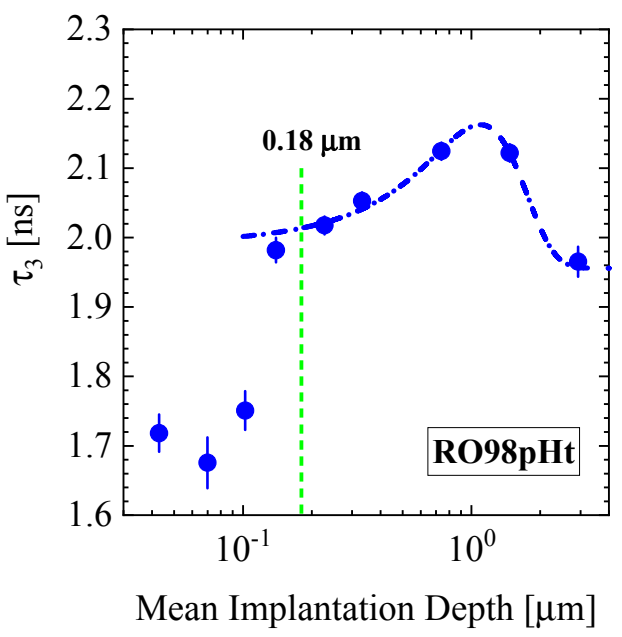

(b)

Figure 9. (a) Intensity of positron lifetimes $\tau_{3}$ and $\tau_{4}$ as well as (b) the lifetime $\tau_{3}$ of the RO98 $\mathrm{pHt}$ membrane versus mean implantation depth. The increases of intensity and lifetime itself are related to larger pore volume fraction and larger volume of pores, respectively. The appearance of the long lifetime $\tau_{4}$ (solid spheres in (a)) indicates a larger mean pore radius in the range of 40-90 A. The green dashed line marks the interface between PA and support layer. 


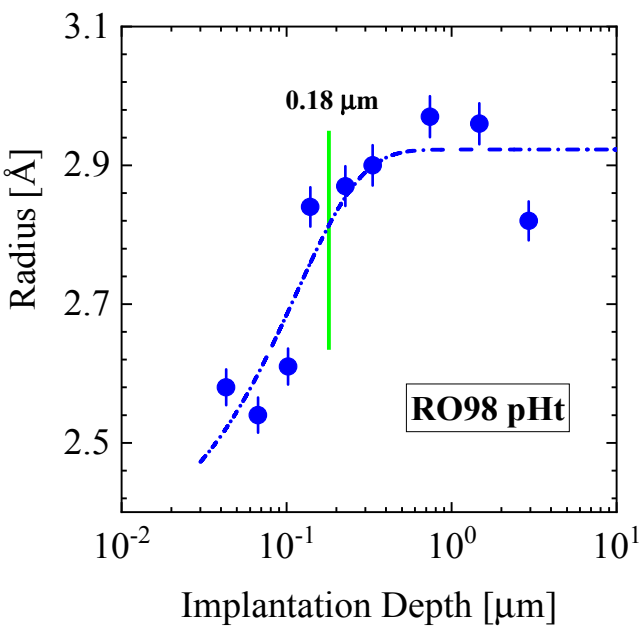

(a)

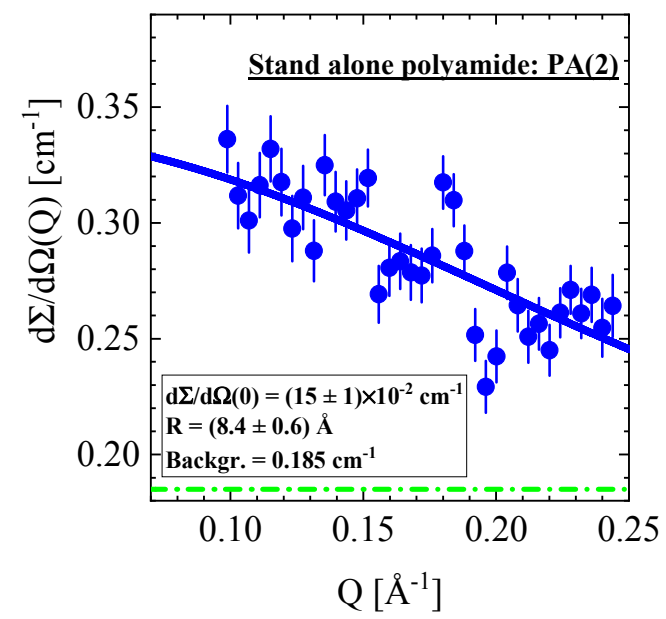

(b)

Figure 10. (a) Radius of pores in the RO98 pHt alfa laval membrane versus implantation depth as determined from $\tau_{3}$ in Figure $9 \mathrm{~b}$. Figure 2a shows the corresponding SANS data at large $Q$ delivering a radius of $(12.4 \pm 0.7) \AA$. (b) For comparison, large $Q$ data from a standalone polyamide layer are depicted delivering a radius of $8.4 \pm 0.6 \AA$ from fitting the form factor of spheres (these data are published in ref [4]). The blue line represents a fit of the data with Equation (A3) and the dashed-dotted line represents the background scattering.

\subsection{Pores of Nanoscale Dimension Determined from PALS and SANS}

\subsubsection{RO98 pHt Membrane}

We compare the results from PALS with the SANS data measured in a Q range between 0.1 and $0.4 \AA^{-1}$. In this range, SANS becomes sensitive to pores of the order $\mathrm{R}=10 \AA$. However, interpretation becomes difficult, as the localization of the scattering centers in the membrane is not possible and incoherent scattering becomes relevant, i.e., $\mathrm{d} \Sigma / \mathrm{d} \Omega_{\text {inc }}$ in Table 2.

Figure 10a shows the pore radius as a function of implantation depth of the cleaned $\mathrm{RO} 98 \mathrm{pHt}$ membrane. The pore radius increases continuously from 2.5 to $2.9 \AA$ in the surface layer depth from 0.02 to $0.18 \mu \mathrm{m}$, before it becomes relatively constant in the measured membrane depth of $2.9 \mu \mathrm{m}$, thereby covering the active polyamide and part of the porous polysulfone support layer.

The corresponding SANS data of the same membrane are shown in Figure 2a, whose large Q data are fitted with the form factor for spheres in Equation (A3) delivering $\mathrm{d} \Sigma / \mathrm{d} \Omega(0)=(0.12 \pm 0.01) \mathrm{cm}^{-1}$ and a $\mathrm{R}=(12.3 \pm 0.7) \AA$, from which we evaluate $\Phi \times \Delta \rho^{2}=(0.15 \pm 0.03) \times 10^{20} \mathrm{~cm}^{-4}$ (Equation (A2)). For comparison, we depict in Figure $10 \mathrm{~b}$ the large $\mathrm{Q}$ data from a standalone polyamide layer published in ref. [4]. The fit using the form factor for spheres delivered $\mathrm{d} \Sigma / \mathrm{d} \Omega(0)=(0.15 \pm 0.01) \mathrm{cm}^{-1}$ and a pore radius of $\mathrm{R}=(8.4 \pm 0.6) \AA$ yield a four times larger $\Phi \times \Delta \rho^{2}=0.60 \times 10^{20} \mathrm{~cm}^{-4}$ and a pore volume fraction $(\Phi)$ of $6.2 \%$. In ref [4], we attributed the larger pore size of SANS to the strong correlation of the pores forming a fractal network structure. The scattering data of the RO98 $\mathrm{pHt}$ membrane and the standalone polyamide layer are in similar range even though the membrane data represent the entire membrane thickness.

\subsubsection{SW30HR, BW30LE, and NF270 Membranes}

In this section, we present PALS and the corresponding SANS data of the SW30HR, BW30LE, and NF270 membranes. Figure 11a shows the PALS data versus implantation depth. The BW membrane shows slightly smaller pores than the SW membrane but otherwise the same shape of increasing radii, which become constant (about $2.9 \AA$ ) beyond the implantation depth of $0.18 \mu \mathrm{m}$ in consistence with the data of the RO98 pHt membrane. 


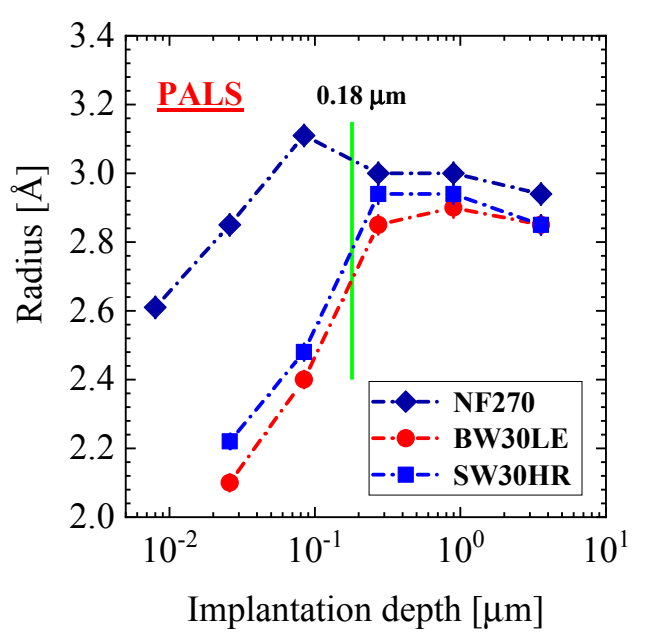

(a)

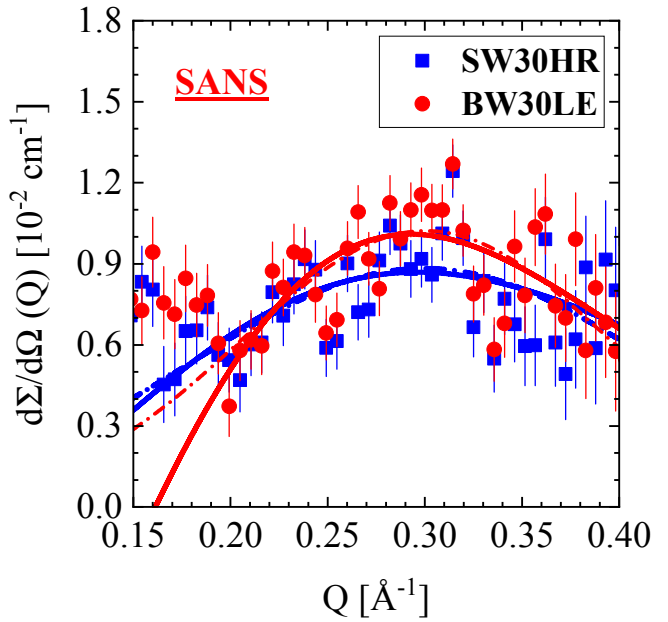

(b)

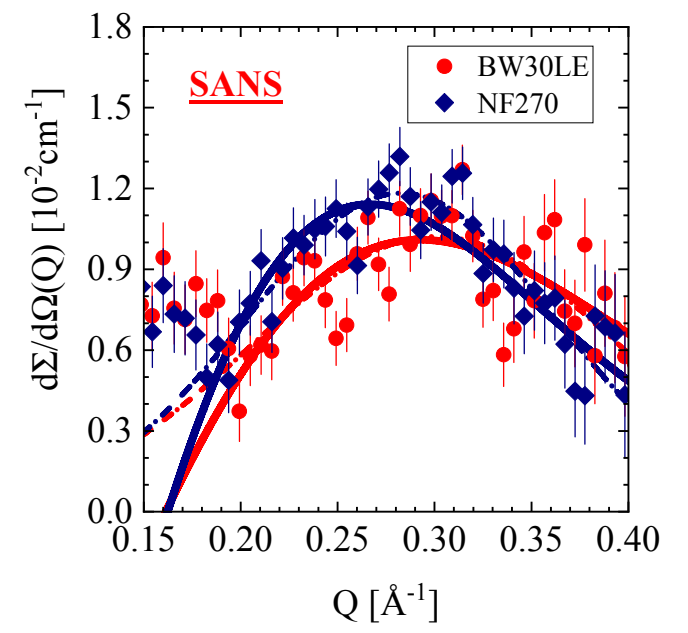

(c)

Figure 11. (a) Radius of the pores versus implantation depths for different membranes as determined with positron-annihilation lifetime spectroscopy (PALS). The symbols cover the error bars. (b,c) Large $Q$ SANS data in vacuum from the same membranes showing an interference peak at $Q_{m}=0.30(0.28) \AA^{-1}$ from densely packed pores. The solid and dashed-dotted curves correspond to fits of the hard-sphere structure factor and Gaussian distribution, respectively.

In this range of implantation, the NF270 membrane also shows pores of increasing, but overall $\sim 30 \%$ larger size, which seems reasonable for a thinner active polyamide layer. Figure $11 \mathrm{~b}, \mathrm{c}$ display in the $Q$ range from 0.15 to $0.40 \AA^{-1}$ the corresponding SANS pattern of the SW30HR, BW30LE and NF270 membranes showing similar sensitivity to micro pores as PALS. For all three membranes, we observe an interference peak at around $0.29 \AA^{-1}$, which corresponds to a periodicity length of the pores of about $25 \AA$ (i.e., $\Lambda_{\mathrm{C}}=1.23 \times\left(2 \pi / \mathrm{Q}_{\mathrm{C}}\right.$ ); (see ref. [37], p. 73). Such an interference peak is not observed for the RO98 pHt membrane (Figure 2a). The scattering patterns in Figure 11 were fitted with two functions. The Gaussian distribution (dashed-dotted lines) according to $\mathrm{d} \Sigma / \mathrm{d} \Omega(\mathrm{Q})=\mathrm{d} \Sigma / \mathrm{d} \Omega\left(\mathrm{Q}_{\mathrm{C}}\right) \times \exp \left(-0.5 \times\left(\left(\mathrm{Q}-\mathrm{Q}_{\mathrm{c}}\right) / \Delta \mathrm{Q}\right)^{2}\right)$ was used for the determination of the peak position and width $\Delta \mathrm{Q}=\left(\mathrm{Q}_{\mathrm{A}}-\mathrm{Q}_{\mathrm{C}}\right)\left(\mathrm{d} \Sigma / \mathrm{d} \Omega\left(\mathrm{Q}_{\mathrm{A}}\right)=\exp (-0.5)=0.607\right)$ and the structure factor was used for concentrated spheres (Equation (A5); solid lines) to determine size (R) and volume fraction $(\Phi)$ of the pores. The correlation length $(\eta)$ of the ordering of pores can be derived from the half-width $\Delta \mathrm{Q}$ of the Gaussian function according to $\eta=\sqrt{1.5} / \Delta \mathrm{Q}$. 
The PALS and SANS parameters are compiled in Table 5. At a membrane depth larger than $0.18 \mu \mathrm{m}$, PALS delivers an average pore radius of $\mathrm{R}=2.9 \AA$ for the $\mathrm{RO}$ and pores only a few percentages larger for the NF membranes in the PA and part of the polysulfone support layers. The fit of the SANS data with the Gaussian function delivers an average distance of the pores of $\Lambda=(26 \pm 1) \AA$, whereas Q2 (Equation (A6)) gives a pore volume fraction between $1.5 \%$ and $1.7 \%$ with respect to the total membrane volume. The fits of the same SANS data with the structure factor of concentrated spheres (Equation (A5)) account for pore radii, which are two to three times larger than the PALS values and show a volume fraction between $14 \%$ and $26 \%$, the larger one for the NF membrane. The latter values of volume fraction were determined from the shape of the structure factor (Equation (A5)) and are thereby independent from absolute calibration of the scattered intensity. A comparison of these values with those from $\mathrm{Q} 2$ analysis allows an estimation of the effective thickness $\left(\mathrm{d}_{\mathrm{eff}}\right)$ of that part of the membrane containing the micro pores. Values of $d_{\text {eff }}=19$ and $12 \mu \mathrm{m}$ were estimated for the SW and BW membranes, whereas a smaller $\mathrm{d}_{\mathrm{eff}}$ of $9 \mu \mathrm{m}$ for the NF membrane.

Table 5. Parameters obtained from PALS and SANS at large Q. The RO98 pHt and Dow-Chem. membranes were measured in $\mathrm{H}_{2} \mathrm{O}$ and air, respectively. Error bars for $\mathrm{R}$ determined by PALS and SANS reflect only statistical errors from the fitting routines.

\begin{tabular}{ccccc}
\hline Parameter & RO98 pHt & SW30HR & BW30LE & NF270 \\
\hline \multicolumn{5}{c}{ PALS } \\
\hline $\mathrm{R}[\AA]$ (at penetration depth $>0.18 \mu \mathrm{m})$ & $2.88 \pm 0.02$ & $2.91 \pm 0.05$ & $2.87 \pm 0.03$ & $2.98 \pm 0.04$ \\
\hline & SANS-Gaussian Distribution & & \\
\hline $\mathrm{Q}_{\mathrm{C}}\left[\AA^{-1}\right]$ & - & $0.30 \pm 0.01$ & $0.30 \pm 0.01$ & $0.28 \pm 0.002$ \\
\hline $\mathrm{Q} 2\left[10^{-4} \mathrm{~cm}^{-1} \AA^{-3}\right]$ & - & 2.37 & 2.28 & 2.02 \\
\hline$\Phi_{\mathrm{Q} 2}[\mathrm{vol} \%]$ & - & 1.72 & 1.66 & 1.47 \\
\hline & $\begin{array}{c}\text { Model of } \\
\text { Spheres }\end{array}$ & Model of Hard-Spheres (Equation (A5)) \\
\hline $\mathrm{d} \Sigma / \mathrm{d} \Omega(0)[10]$ & $12 \pm 1$ & $2.08 \pm 0.10$ & $2.64 \pm 0.15$ & $3.1 \pm 0.1$ \\
\hline $\mathrm{R}[\AA]$ & $12.3 \pm 0.7$ & $6.1 \pm 0.5$ & $6.8 \pm 0.4$ & $6.1 \pm 0.2$ \\
\hline$\Phi_{0}[\%]$ & - & $14 \pm 2$ & $21 \pm 4$ & $26 \pm 2$ \\
\hline $\mathrm{Q}^{2}\left[10^{-4} \mathrm{~cm}^{-1} \AA^{-3}\right]$ & 2.33 & 2.6 & 2.53 & 2.3 \\
\hline$\Phi_{\mathrm{Q} 2}[\mathrm{vol} \%]$ & $0.9-1.1$ & 1.9 & 1.84 & 1.66 \\
\hline $\mathrm{d}_{\mathrm{eff}}[\mu \mathrm{m}]$ & - & 19 & 12 & 9 \\
\hline
\end{tabular}

Several PALS studies show pores of similar radii in the PA layer. For instance, ref. [38] states that "commercially available RO membranes have a mean free-volume hole-radius of 2.0-2.9 $\AA$ in the active skin layer with the thickness of approximately $1000 \AA$ ". In another study [39], it is determined by PALS that" the thin films of cross-linked aromatic polyamide RO membranes are composed of two types of pores having radii of about 2.1-2.4 $\AA$ from $\tau_{3}$ and 3.5-4.5 $\AA$ from $\tau_{4}$ ". Fujioka et al. [40] found that ESPA2 and ESPAB membranes have the same mean free-volume hole-radii of $2.89 \AA$, while that for SWC5 membrane was determined to be $2.59 \AA$. It was found that these numbers correlate with the rejection properties of the corresponding membranes.

Our PALS data show a gradual increase in pore size from about 2.1 to 2.8 and from 2.6 to $3.1 \AA$ in a thin surface layer of $0.18 \mu \mathrm{m}$ for the RO and NF membranes, respectively (Figures 10a and 11a). To our knowledge, such a behavior of pore size is visualized here the first time for a wider set of commercial TFC membranes and seems to display a general property of TFC membranes. We have no adequate explanation. Nevertheless, the following discussion might shed some light on this phenomenon. As the PA skin and the PSU (PES) porous support layers are in glass-like states (see $\mathrm{T}_{\mathrm{G}}$ in Table 2), we may consider the surface effects of polymer glasses. Physical aging, i.e., $\beta$ relaxation was studied 
in poly(methyl methacrylate) (PMMA) glass in bulk and at the surface in ref. [41]. Physical aging drives glasses in the direction of larger mass density in accordance with the equilibrium state of the corresponding melt. The authors observed a reduced relaxation rate within a range of $\sim 0.25 \mu \mathrm{m}$ from the surface inward, becoming about two times smaller than the bulk relaxation rate. The reason for this behavior is still unclear according to the authors. However, there might be some correlation of continuously declining pore size towards the surface, accompanied with larger polymer mass density and the decline of the physical relaxation rate.

Our last comment refers to the size and volume fraction of $\AA$ large pores. Whereas PALS shows almost no variation of pore size for the membranes in Table 5, SANS shows a larger variation. Figure $11 \mathrm{~b}, \mathrm{c}$ shows for the BW30LE membrane, respectively, a $10 \%$ and $\sim 50 \%$ larger pore radius and volume fraction, whereas for the NF270 membrane, the same pore radius but a $\sim 86 \%$ larger volume fraction in comparison with the SW30HR membrane (Table 5).

\subsubsection{TM820 Seawater RO Membrane, Pristine and Scaled}

Finally, we present the large Q SANS data from the TM820 membrane in non-scaled and scaled conditions (see Table 1, Section 2.1 as well as Figure 12 and Table 6). A comparison of these data seems interesting as scaling shows a strong effect on micro pores, but no effect on the $\mu \mathrm{m}$ large pores, as the low $\mathrm{Q}$ data (not shown) do not show any visible effect from scaling. Pores of the radius of $1.8 \AA$ and $0.9 \AA$ were determined from PALS as compiled in Table 6, whereas SANS delivers densely packed pores of $(7.6 \pm 0.8) \AA$ and $(6.8 \pm 2.6) \AA$ radius distributed at the average distances of $\Lambda=(30 \pm 1.2)$ $\AA$ and $(25.8 \pm 0.9) \AA$ in the unscaled and scaled membranes, respectively. The volume fraction of the pores in both membranes is about $18 \%$ and distributed in a $\sim 16 \mu \mathrm{m}$ thick layer of the membrane. It seems remarkable that scaling reduces the pore radii by a factor of two (PALS), whereas the SANS pore radius of the scaled membrane also seemed declined, however, this could only be determined with large error, whereas the volume fraction of pores declined between $10 \%$ and $20 \%$ as evaluated from Q2.

Table 6. Parameters of non-scaled and scaled TM820 membranes determined from PALS and SANS at large Q.

\begin{tabular}{ccc}
\hline Parameter & Pristine Membrane & Scaled Membrane \\
\hline & PALS \\
\hline $\mathrm{R}[\AA]$ & $1.82 \pm 0.41$ & $0.93 \pm 0.84$ \\
\hline $\mathrm{Q}_{\mathrm{C}}\left[\AA^{-1}\right]$ & SANS—Gaussian Distribution \\
\hline $\mathrm{Q} 2\left[10^{-4} \mathrm{~cm}^{-1} \AA^{-3}\right]$ & $0.26 \pm 0.01$ & $0.30 \pm 0.01$ \\
\hline & 2.69 & 2.16 \\
\hline $\mathrm{d} \Sigma / \mathrm{d} \Omega(0)[10]$ & SANS-Model of hard-spheres \\
\hline $\mathrm{R}[\AA]$ & $3.3 \pm 0.2$ & $2.0 \pm 0.6$ \\
\hline$\Phi_{0}[\mathrm{vol} \%]$ & $7.6 \pm 0.8$ & $6.8 \pm 2.6$ \\
\hline $\mathrm{Q} 2\left[10^{-4} \mathrm{~cm}^{-1} \AA^{-3}\right]$ & $18 \pm 4$ & 2.50 \\
\hline$\Phi_{\mathrm{Q} 2}[\mathrm{vol} \%]$ & 2.78 & 1.82 \\
\hline $\mathrm{d}_{\mathrm{eff}}[\mu \mathrm{m}]$ & 2.02 & $\sim 14$ \\
\hline
\end{tabular}




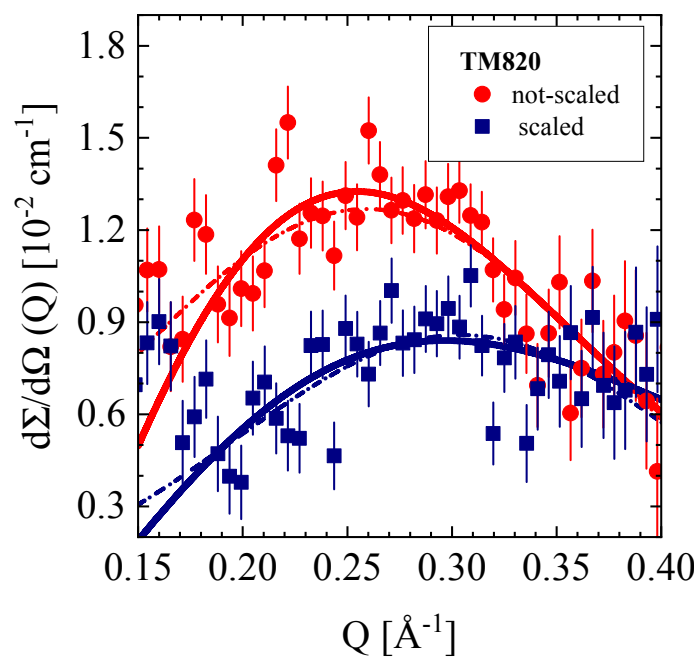

Figure 12. The hard-sphere model (solid lines) as well as the Gaussian distribution (dashed-dotted lines) describe the scattering from small correlated units, probably pores (Toray membrane company). Background scattering of $0.1 \mathrm{~cm}^{-1}$ was subtracted.

\section{Conclusions}

In this manuscript, we reported about the morphology of several commercial RO membranes and a NF membrane from the perspective of SANS and PALS. Both techniques are complementary: PALS determines the depth profile of pores of radii of several $\AA$, which are distributed over a distance of about $3 \mu \mathrm{m}$ from the membrane surface. This includes the PA selective layer and the underneath supporting layer. SANS, on the other hand, allows identification of the membrane polymers and determination of pores of sizes from a few $\AA$ to $\mu \mathrm{m}$ dimension distributed over the entire membrane thickness. Identification of the membrane polymers and thereby porous support and nonwoven fabric layers of thin film composite (TFC), RO, and NF membranes needs the application of contrast variation experiments, in which the minima of the scattering length density $\left(\propto \rho_{S}^{2}\right)$ identifies the polymer species via comparing with $\rho$ of the polymers in Table 2 . In this case, the scattered intensity should be zero, as observed for the polysulfone but not for the polyester layer, because of its internal heterogeneities not accessible to the contrast media.

The polyamide skin layer of RO membranes is too thin to be detected at small $Q$ by SANS. An independent SANS and PALS exploration of a standalone PA layer in ref. [4] delivered a network structure of pores of $\sim 8 \AA$ radius. Pores of similar size (Tables 5 and 6 ) were detected for all membranes at $Q>0.1 \AA^{-1}$, which might be allocated to the skin as well as to the porous support layer. On the other hand, PALS was able to detect pores with a radius of nearly $3 \AA$ over a distance of nearly $4 \mu \mathrm{m}$ from the membrane surface, thereby covering the active polyamide skin layer and part of the polysulfone porous support layer. The size of the pores seems to be unaffected by the transition from the polyamide to the polysulfone layer. An interesting observation is the declining pore size, up to $40 \%$ in a $\sim 0.18 \mu \mathrm{m}$ thick surface layer. All RO membranes depicted in Figures 10a and 11a show similar shrinking within $\sim 0.2 \mu \mathrm{m}$ the same radius for pores, whereas the NF270 membrane in Figure 11a shows a smaller decline of less than $20 \%$. This observation seems to be correlated with the glass state of the polymers, showing a reduced structural relaxation due to larger mass density at the surface layer, which is of similar thickness as outlined in ref. [41]. This correlation might be a valuable hint for better understanding surface effects in polymer glasses.

Freger [42] discusses an inhomogeneous mass distribution across PA skin layers of the RO membrane. He argues that PA skin layers are composed of negatively fixed and positively charged layers with an intermediate layer of larger polymer density acting as the actual selective barrier. As shown in [42] (Figure 6), this selective barrier is covered by a charged layer of $\sim 1 \mu \mathrm{m}$ forming the 
outer interface of the polyamide layer. Apart from a thickness of the PA layer that is too large, these calculations do not correlate with our PALS observation in Figures 10 and 11a.

Pore radii in the PA membrane layer and the adjacent support layer as determined by PALS and SANS seem to differ as much as a factor of $2-3$. The presented SANS radii are an average over eventual asymmetric pore dimensions. Furthermore, neglecting magnetic scattering, neutron scattering "sees" the nuclei of the surrounding matter. On the contrary, in PALS, the o-Ps looks for the nearest electron and thereby is sensitive to the shortest distance in the pore. Furthermore, Equation (A11) contains two different radii, the shorter hard-core radius $\mathrm{R}$, assuming zero electron density and a shell of thickness $\Delta \mathrm{R}=\mathrm{R}_{0}-\mathrm{R}=1.656 \AA$ to account for the increasing electron density towards the surface of the pore. The shorter radius $(R)$ has been noted throughout this paper. Taking $R_{o}$ as the pore radius instead, an average PALS radius of $R_{\text {PALS }}=4.5 \AA$ is yielded at penetration depths of $>1.8 \mu \mathrm{m}$. This $R_{\text {PALS }}$ is about $70 \%$ of the value determined by SANS, i.e., $R_{\text {SANS }}=6.1-6.8 \AA$. Taking into account that PALS "sees" the shortest distance within a pore, and SANS averages over all distances in a pore, both methods give very similar pore radii. A further aspect to be considered is the network structure of $R<10 \AA$ pores predicted in [36] and observed with SANS/PALS in [4], which appears relevant for the transport of water.

The polymers of the porous support and the fibers of the nonwoven fabric layers could be identified via contrast variation measurements. In the case of the porous support layer, polysulfone (PSU) and polyethersulfone (PES) polymers were identified, as well as open pores between 0.3 and 0.6 $\mu \mathrm{m}$ radius of a volume fraction of $\Phi \cong 4.5 \mathrm{vol} \%$ accessible for the contrast media. The volume fraction of closed pores, inaccessible for the contrast medium, is less than $\Phi=6 \mathrm{vol} \%$ except for the XLE membrane, showing a much larger $\Phi \cong 50 \mathrm{vol} \%$. Graft polymerization by MA on the XLE membrane shows a decline of accessible pore volume fraction due to the MA polymerization inside the pores, which decreases the volume of pores accessible for liquids.

Segments of the fibers of the nonwoven fabric layer determine scattering, because of loose packing to a network as visualized in Figure $8 \mathrm{~b}$. Radii of gyration between 1 and $1.3 \mu \mathrm{m}$ of polypropylene (PP) and polyethylenterephthalat (PET) between $2 \mathrm{vol} \%$ and $4 \mathrm{vol} \%$ were identified. Smaller fibers were observed for the XLE membranes whose chemistry could be explained by a mixture of $73 \mathrm{vol}$ $\%$ PET and $23 \mathrm{vol} \%$ of low density polyethylene (PE). The latter observation is consistent with its large scattering from the internal structure of the fiber prepared from a mixture of two polymers. The other two membranes (SW30HR and BW30LE) show nearly 20\% contribution from the internal structure of the fibers, which we interpret as from pores. The glass state of PET fibers may have some influence on scattering due to the internal structure, whereas polypropylene was in a condition of melt $\left(\mathrm{T}_{\mathrm{G}}=-10^{\circ} \mathrm{C}\right)$, which, however, cannot be tested with SANS due to its negative scattering length density (Figure 4c,d).

Author Contributions: Conceptualization: D.S., W.P., R.K., Y.O.; Experimental investigation (SANS), V.P., D.S., H.F.; (PALS), M.D., C.H.; Membranes, R.K. and Y.O.; writing and editing, D.S., C.H., Y.O., W.P. All authors have read and agreed to the published version of the manuscript.

Funding: This work was funded by the German-Israeli Foundation for Scientific Research and Development (GIF), grant No: I-101-307.4-2013.

Acknowledgments: SANS and PALS experiments were performed at, respectively, the KWS-1, 3 and Nepomuc instruments at the Heinz Maier-Leibnitz Zentrum (MLZ), Garching, Germany. We would like to thank Stephan Wild and Dipl.W.-Ing Micha Kruse from Alfa Laval for providing the RO98 pHt membrane. D.S. would like to thank Prof. Dr. Stephan Förster, head of the Jülich Centre for Neutron Science (JCNS-1) and Institute for Complex systems (ICS-1) at the Forschungszentrum Jülich $\mathrm{GmbH}$ for his hospitality.

Conflicts of Interest: The authors declare no conflict of interest.

\section{Appendix A.}

In Appendices A.1 and A.2 we provide the scattering laws applied for the analysis of the SANS scattering data, as well as an explanation of SANS contrast variation together with the scattering length 
densities of the contrast media $\mathrm{H}_{2} \mathrm{O} / \mathrm{D}_{2} \mathrm{O}$, and the $\mathrm{SCFs} \mathrm{CO}_{2}$, and $\mathrm{CD}_{4}$, in Appendix A.3. Appendix A.4 outlines PALS in polymers.

\section{Appendix A.1. SANS Scattering Laws}

Scattering techniques using neutrons and photons are well established tools both from theoretical and experimental aspects [26] (p. 188). Scattering originates from domains that differ from their surroundings in their chemical composition and/or mass density. In our case, pores, or even segments of polymer fibers represent those domains. SANS is a quantitative method, which determines structural parameters, which are averaged over macroscopic large volumes of the order of $0.1 \mathrm{~cm}^{3}$. In this respect SANS is complementary to TEM, which makes individual domains visible.

The SANS pattern of the full membrane represents a linear superposition of scattering from all three layers weighted with the ratio of thickness according to $\mathrm{d}_{\mathrm{i}} / \mathrm{d}_{\text {tot }}$ with " $\mathrm{i}$ " and "tot" indicating the polyamide, polysulfone, or polyester/polypropylene layer and the total thickness of the membrane, respectively. The differences in chemical composition and/or mass density are contained in the scattering contrast, which for neutrons is determined by the difference of $\rho$ from the domain $(P)$ and its surrounding (S) squared, i.e., $\Delta \rho_{i}^{2}=\left(\rho_{P, i}-\rho_{S}\right)^{2}$. The scattering length densities of the relevant polymers are compiled in Table 2. The scattered neutrons intensity of layer " $\mathrm{i}$ " is expressed in Equation (A1) as differential macroscopic cross-section $\left(\mathrm{d} \Sigma / \mathrm{d} \Omega_{\mathrm{i}}(\mathrm{Q})\right)$ determined

$$
\mathrm{d} \Sigma / \mathrm{d} \Omega_{\mathrm{i}}(\mathrm{Q})=\mathrm{d} \Sigma / \mathrm{d} \Omega_{\mathrm{i}}(0) \times \mathrm{F}_{\mathrm{i}}(\mathrm{Q}) \times \mathrm{S}_{\mathrm{i}}(\mathrm{Q})
$$

in units of $\mathrm{cm}^{-1}$ (i.e., scattering per $\mathrm{cm}^{3}$ of sample volume) as product of the form factor $\mathrm{F}_{\mathrm{i}}(\mathrm{Q})\left(\mathrm{F}_{\mathrm{i}}(0)=\right.$ 1), structure factor $S_{i}(Q)\left(S_{i}(Q \rightarrow \infty)=1\right)$, and macroscopic cross-section at $Q=0$, which according to Equation $(\mathrm{A} 2)$, is determined by the domain volume $\left(\mathrm{V}_{\mathrm{P}}\right)$, domain volume fraction $\left(\Phi_{\mathrm{P}}\right)$ of

$$
\mathrm{d} \Sigma / \mathrm{d} \Omega_{i}(\mathrm{Q}=0)=\Phi_{\mathrm{P}, \mathrm{i}} \mathrm{V}_{\mathrm{P}, \mathrm{i}}\left[\rho_{\mathrm{P}, \mathrm{i}}-\rho_{\mathrm{S}}\right]^{2}
$$

domains such as pores or fibers in layer $i$, and the scattering contrast $\left[\rho_{P, i}-\rho_{S}\right]^{2}$ compiled for relevant polymers of TFC membranes in Table 2. In cases of $\mu \mathrm{m}$ large pores we apply the form factor of spherically shaped domains of radius R showing the well-known expression in Equation (A3) [26,37], whereas for the larger pores we apply Beaucage's equation (Equation (A4)) for

$$
\mathrm{F}(\mathrm{Q})=\frac{9\left(\sin \left(\mathrm{QR}_{\mathrm{P}}\right)-\mathrm{QR}_{\mathrm{P}} \cos \left(\mathrm{QR}_{\mathrm{P}}\right)\right)^{2}}{\left(\mathrm{QR}_{\mathrm{P}}\right)^{6}}
$$

quantitative analyses of the scattering pattern describing the data at $\mathrm{Q}<1 / \mathrm{R}_{\mathrm{g}}$ by Guinier's approximation (first term) and at $\mathrm{Q}>1 / \mathrm{R}_{\mathrm{g}}$ by a power law $\left(\mathrm{P}_{\mathrm{P}} \mathrm{Q}^{-\alpha}\right)$ with the exponent $\alpha_{\mathrm{i}}$ and the

$$
\mathrm{d} \Sigma / \mathrm{d} \Omega_{\mathrm{i}}(\mathrm{Q})=\mathrm{d} \Sigma / \mathrm{d} \Omega_{\mathrm{i}}(0) \exp \left(-\mathrm{u}_{\mathrm{i}}^{2} / 3\right)+\mathrm{P}_{\mathrm{P}_{\mathrm{i}}}\left[\left(\operatorname{erf}\left(\mathrm{u}_{\mathrm{i}}^{2} / \sqrt{6}\right)\right)^{3} / \mathrm{Q}\right]^{\alpha_{\mathrm{i}}}
$$

Amplitude $P_{\mathrm{P}}$ (second term) [43]. The radius of gyration $\mathrm{R}_{\mathrm{g}}$ is defined according to $\mathrm{R}_{\mathrm{g}}^{2}=$ $\int \mathbf{r}^{2} \rho(\mathbf{r}) \mathrm{d} \mathbf{r} / \int \rho(\mathbf{r}) \mathrm{d} \mathbf{r}$ as second moment of the scattering length density $\rho(\mathbf{r})$ being a function of the local vector $\mathbf{r}$. [26] ( $\mathrm{p}$ 158). The parameter $u_{i}$ expresses the product of $Q$ and $R_{g, i}$.

The scattering law in Equation (A4) is valid in the limit of low particle concentration when scattering is determined from individual domains $S(Q)=1$, i.e., there is no correlation between the particles. Scattering of higher particle concentration shows correlations such as those observed for 
micro pores in the polyamide layer formed as fractal structure [4] or, as shown in Section 3.4, for TFC membranes. The analytical expression of $\mathrm{S}(\mathrm{Q})$ in Equation (A5) describes an ensemble of

$$
\mathrm{S}_{\mathrm{HS}}(\mathrm{Q})=1-8 \Phi \frac{3(\sin 2 \mathrm{QR}-2 \mathrm{QR} \cos 2 \mathrm{QR})}{(2 \mathrm{QR})^{3}}
$$

concentrated spheres interacting via their excluded volume [26] (p. 172). The parameters, $\Phi$ and $R$, are volume fraction and radius of the spheres, respectively.

An important parameter is the second moment, $Q 2=\int \mathrm{Q}^{2} \mathrm{~d} \Sigma / \mathrm{d} \Omega(\mathrm{Q}) \mathrm{dQ}$, sometimes called the "invariant" of scattering of isotropic scattering centers, which according to Equation (A6) is determined only from the product of particle volume fraction and scattering contrast of the

$$
\mathrm{Q} 2_{i}=2 \pi^{2} \Phi_{i}\left(1-\Phi_{i}\right)\left[\rho_{P, i}-\rho_{\mathrm{S}}\right]^{2}
$$

corresponding layer but does not depends on domain size [26].

\section{Appendix A.2. Correction for Multiple Scattering}

Correction for multiple scattering becomes relevant in case of low transmission of $\mathrm{T}<0.3$ in the classical SANS Q-range $\left(Q>0.001 \AA^{-1}\right)$ or $T<0.8$ for structures of $\mu \mathrm{m}$ in size measured in the extended Q-range of smaller than $\mathrm{Q} \approx 10^{-4} \AA^{-1}$ as measured with VSANS. A theoretical summary of multiple scattering has been given in refs. [44], which is based on original considerations by Schelten et. al. [45] (see also [46]). The numerical program for the calculus is found in ref. [47]. The essential formula for considering multiple scattering effects, Equation (A7), contains sample thickness $d$, neutron wavelength $\lambda$, and the apparently measured and corrected

$$
\mathrm{d} \widetilde{\Sigma}_{\text {corr }} / \mathrm{d} \Omega(\mathrm{r})=2 \pi /\left(\mathrm{d} \times \lambda^{2}\right) \times \ln \left[\mathrm{d} \times \lambda^{2} /(2 \pi) \times \mathrm{d} \widetilde{\Sigma}_{\text {app }} / \mathrm{d} \Omega(\mathrm{r})+1\right]
$$

macroscopic cross sections $\mathrm{d} \widetilde{\Sigma}_{\text {app }} / \mathrm{d} \Omega(\mathrm{r})$ and $\mathrm{d} \widetilde{\Sigma}_{\text {corr }} / \mathrm{d} \Omega(\mathrm{r})$, respectively. The latter two are given in 'real' space connected via a Hankel transformation, i.e., for an arbitrary function $\mathrm{a}(\mathrm{Q})$ and the

$$
\mathrm{a}(\mathrm{r})=\int_{0}^{\infty} \mathrm{a}(\mathrm{Q}) \cdot \mathrm{Q} \cdot \mathrm{J}_{0}(\mathrm{Qr}) \mathrm{dQ}
$$

Bessel function of zero order $\mathrm{J}_{0}$, if the scattering is isotropic. In practical terms, two transformations are necessary, first towards real space, deconvolution according to Equation (A7) and one back in reciprocal space to display the corrected scattering cross-section. Other proposed calibrations involve the explicit knowledge of the macroscopic cross sections for the incoherent scattering and the absorption. Practically, these methods did not provide better or more reliable results. For the proposed correction method here (Equation (A7)), this means that the apparent cross-section needs to be reliably calibrated. The Taylor expansion of Equation (A7) for small apparent macroscopic cross sections (i.e., $\ln (1+\varepsilon)=$ $\varepsilon-\varepsilon^{2} / 2+\varepsilon^{3} / 3-\ldots$ ) leads to degree wise corrections of multiple scattering corrections (i.e., 1, 2, 3 times), but they are not considered any further here.

\section{Appendix A.3. The Importance of the Contrast Media for SANS Evaluation of TFC Membranes}

The use of different contrast media for SANS makes it possible to identify scattering from the supporting layers of TFC membranes. In practice, the membranes are exposed to a series of solvents, whose scattering contrast, $\Delta \rho^{2}=\left[\rho_{\mathrm{P}}-\rho_{\mathrm{S}}\right]^{2}$ can be changed over a wide range. The goal is to find the proper solvent conditions from which the matching condition, namely $\mathrm{d} \Sigma / \mathrm{d} \Omega(\mathrm{Q}) \propto \Delta \rho^{2}=0$ at $\rho_{\mathrm{P}}=\rho_{\mathrm{S}}$ (Equations (A1) and (A2)), can be determined, i.e., the scattering length density $\rho_{P}$ of the polymer. For 
the identification of the polymer, the experimental $\rho_{\mathrm{P}}$ has to be compared with the calculated $\rho$ in Table 2.

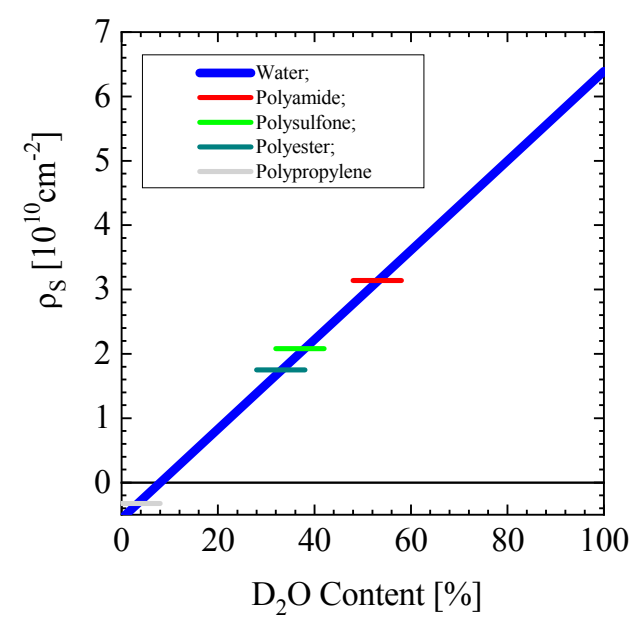

Figure A1. Coherent scattering length density of the $\mathrm{H}_{2} \mathrm{O} / \mathrm{D}_{2} \mathrm{O}$ mixture according to $\rho=()-0.561+$ $6.933 \times \Phi_{\mathrm{D}_{2} \mathrm{O}} \times 10^{10} \mathrm{~cm}^{-2}$ as function of $\mathrm{D}_{2} \mathrm{O}$ volume fraction. The coherent scattering length of the RO-membrane polymeric components are marked as short lines indicating the corresponding matching conditions.

Well-known and often used contrast mediums are mixtures of $\mathrm{H}_{2} \mathrm{O}$ and $\mathrm{D}_{2} \mathrm{O}$ at different ratios. Figure $1 \mathrm{~A}$ shows the average coherent scattering length density $\left(\rho_{\mathrm{S}}\right)$ of water molecules versus $\mathrm{D}_{2} \mathrm{O}$ concentration in $\mathrm{H}_{2} \mathrm{O}$. It is evident that there is a continuous change of $\rho_{\mathrm{S}}$ from -0.561 to $6.37210^{10}$ $\mathrm{cm}^{-2}$. The isotope effect on scattering is explained by the interaction of neutrons with the nucleus of atoms thereby delivering individual values for the coherent scattering length for isotopes of the same element as tabulated in [15]. The scattering length densities of the polymers in Table 2 are determined from the atomic coherent scattering length and monomer molar volume according to $\rho=\sum_{j} b_{j} / \Omega_{\mathrm{M}}$.

SCFs are less known as contrast media in SANS, i.e., fluids at temperatures and pressures above the critical point. These media have the important advantage of only needing one membrane and $\rho_{S}$ can be sensitively tuned by pressure. This is in contrast to $\mathrm{H}_{2} \mathrm{O} / \mathrm{D}_{2} \mathrm{O}$, in which one needs a new membrane for each mixture, which is much more time consuming and prone to errors.

In this study, we used carbon dioxide $\left(\mathrm{CO}_{2}\right)$ and deuterated methane $\left(\mathrm{CD}_{4}\right)$ as contrast media. Figure A2a,b show the isotherms of $\rho_{\mathrm{S}}$ for $\mathrm{CO}_{2}$ at $10,23.8$ and $38^{\circ} \mathrm{C}$ and $\mathrm{CD}_{4}$ for $10{ }^{\circ} \mathrm{C}$ for pressures between 1 and 500 bar. A recent SANS study, with $\mathrm{CO}_{2}$ as SCF at $45^{\circ} \mathrm{C}$, shows the effect of pressure on the thermal number density fluctuations [48]. In this reference, we also describe the equipment of the pressure cell used for this experiment. For $\mathrm{CO}_{2}$ at $10^{\circ} \mathrm{C}$ and $23.8{ }^{\circ} \mathrm{C}$ a first order gas/liquid phase transition is seen from the jump of $\rho$ at $\mathrm{P}=62.5 \mathrm{bar}$ and $45.1 \mathrm{bar}$, respectively, whereas the other two isotherms show $\rho_{\mathrm{S}}$ beyond the critical point, i.e., in the SCF-regime beyond $31^{\circ} \mathrm{C} / 73.8$ bar and $-82.6^{\circ} \mathrm{C} / 45.9$ bar for $\mathrm{CO}_{2}$ and $\mathrm{CH}_{4}$, respectively. This indicates a transition of the gases from low pressure to SCF at higher pressure, thereby enabling a gradual increase of $\rho_{\mathrm{S}}$ by varying the pressure where $\mathrm{CO}_{2}$ and $\mathrm{CD}_{4}$ cover a large range of values for $\rho_{\mathrm{S}}$ up to 500 bar. On the other hand, $\mathrm{CH}_{4}$ cannot work as contrast medium because its $\rho_{\mathrm{S}}$ is negative due to the negative coherent scattering length of the hydrogen $\left(b_{\mathrm{H}}=-3.739 \times 10^{-13} \mathrm{~cm}\right)[15]$. 


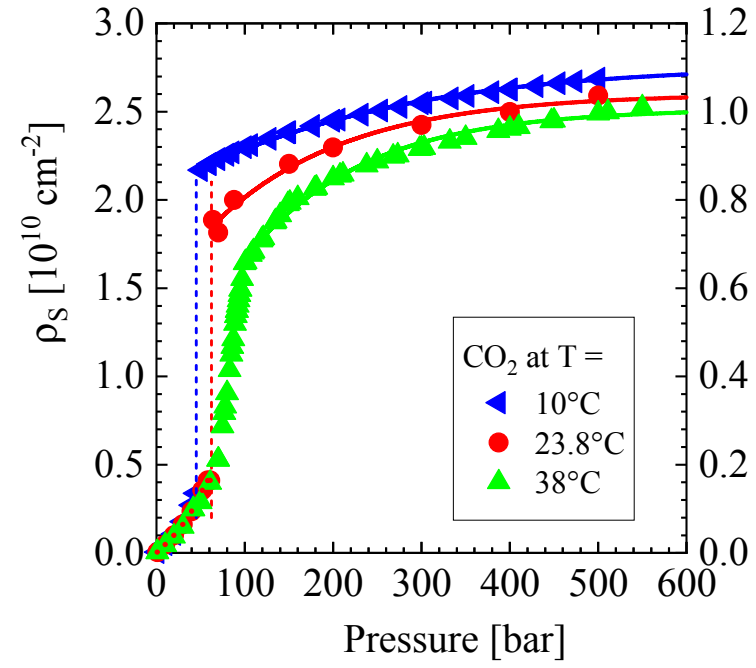

(a)

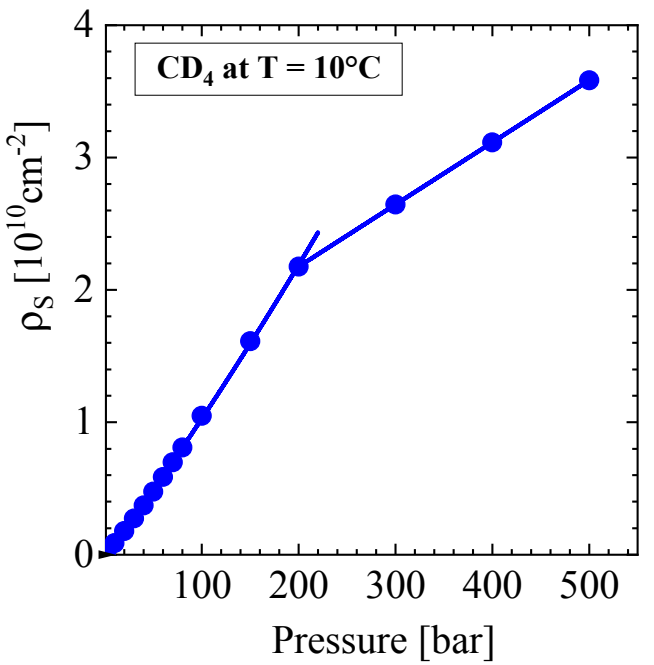

(b)

Figure A2. Scattering length density of $\mathrm{CO}_{2}(\mathbf{a})$ and $\mathrm{CD}_{4}(\mathbf{b})$ as calculated from scattering length and number density $[15,24] . \mathrm{CO}_{2}$ and $\mathrm{CD}_{4}$ become supercritical above their critical temperature and pressure at $31^{\circ} \mathrm{C} / 73.8$ bar and $-82.6^{\circ} \mathrm{C} / 45.9$ bar, respectively.

Ideally, the scattering of homogenous polymeric materials should become zero at the matching condition when the contrast medium is filling all membrane pores. However, such condition is seldom fulfilled in RO membranes, thereby showing finite scattering at the minima of the matching scattering length density, $\rho_{\mathrm{m}}$. Furthermore, $\mathrm{R}_{\mathrm{g}}$ changes with $\rho_{\mathrm{S}}$ showing as for the SW30HR and BW30LE membranes a maximum at the matching condition $\left(\rho_{\mathrm{m}}\right)$ (Figures $5 \mathrm{~b}$ and $6 \mathrm{~b}$ ). The expression of the experimental $R_{\mathrm{g}}$ in Equation (A9) is described as a function of $\rho_{\mathrm{S}}$ [37],

$$
\mathrm{R}_{\mathrm{g}}^{2}\left(\rho_{\mathrm{S}}\right)=\left[\mathrm{I}_{\mathrm{cl}}(0) \times \mathrm{R}_{\mathrm{g}, \mathrm{cl}}^{2} \times \rho_{\mathrm{P}}^{2}+\mathrm{I}_{\mathrm{op}}(0) \times \mathrm{R}_{\mathrm{g}, \mathrm{op}}^{2} \times\left(\rho_{\mathrm{P}}-\rho_{\mathrm{S}}\right)^{2}\right] /\left[\mathrm{I}_{\mathrm{cl}}(0) \times \rho_{\mathrm{P}}^{2}+\mathrm{I}_{\mathrm{op}}(0) \times\left(\rho_{\mathrm{P}}-\rho_{\mathrm{S}}\right)^{2}\right]
$$

$\mathrm{I}_{\mathrm{A}}(0)=\mathrm{d} \Sigma / \mathrm{d} \Omega_{\mathrm{A}}(0) /\left(\rho_{\mathrm{A}}-\rho_{\mathrm{S}}\right)^{2}$ as well as the $\mathrm{R}_{\mathrm{g}}$ 's of the open and closed pores. Equation (A9) is derived from the sum of scattering of the two pore contributions in Equation (A10), both

$$
\mathrm{d} \Sigma / \mathrm{d} \Omega_{\mathrm{A}}(\mathrm{Q}) \cong \mathrm{d} \Sigma / \mathrm{d} \Omega_{\mathrm{A}}(0)\left[1+\mathrm{R}_{\mathrm{A}}^{2} \mathrm{Q}^{2} / 3\right] \operatorname{and} \mathrm{d} \Sigma / \mathrm{d} \Omega_{\mathrm{A}}(\mathrm{Q})=\mathrm{d} \Sigma / \mathrm{d} \Omega_{\mathrm{op}}(\mathrm{Q})+\mathrm{d} \Sigma / \mathrm{d} \Omega_{\mathrm{cl}}(\mathrm{Q})
$$

approximated by the first two terms of the Taylor expansion of Guinier's law [26] (p167)). In vacuum, i.e., $\rho_{\mathrm{S}}=0$ one gets: with $\gamma=\mathrm{I}_{\mathrm{cl}}(0) /\left[\mathrm{I}_{\mathrm{cl}}(0)+\mathrm{I}_{\mathrm{op}}(0)\right]$. The subscripts "op" (open) stands for pores accessible for fluids in homogenous polymeric material, and therefore: $d \Sigma / d \Omega_{\mathrm{OP}} \propto\left(\rho_{\mathrm{P}}-\rho_{\mathrm{S}}\right)^{2}$, whereas " $\mathrm{cl}^{\prime}$ (closed) arises from scattering of an internal inhomogeneity whose $\mathrm{R}_{\mathrm{g}}$ is independent of $\rho_{\mathrm{S}}$, i.e., $\mathrm{d} \Sigma / \mathrm{d} \Omega_{\mathrm{cl}}(0) \propto\left(\rho_{\mathrm{P} 1}-\rho_{\mathrm{P} 2}\right)^{2}$. For closed pores $\mathrm{d} \Sigma / \mathrm{d} \Omega_{\mathrm{cl}}(0) \propto \rho_{\mathrm{P}}{ }^{2}$. The two radii $\mathrm{R}_{\mathrm{g}, \mathrm{op}}$ and $\mathrm{R}_{\mathrm{g}, \mathrm{cl}}$ are determined from $R_{g}$ at the contrasts $\rho_{S}=0$ and $\rho_{S}=\rho_{P}$, i.e., for vacuum and matching condition, respectively.

\section{Appendix A.4. PALS in Polymers}

In polymers, the lifetime of ortho-Positronium (o-Ps), a bound state of positron and electron, is correlated with the void size where o-Ps annihilates the electrons at the void wall by interaction (pick-off process). The so-called Tao-Eldrup model (e.g., ref. [49] (Appendix)) describes the relation between the lifetime $(\tau)$ of o-Ps, which is assumed to be in its ground state, and the void size described by a sphere with radius $R_{0}$. A smaller radius $R$ is defined as a kind of hard sphere radius, whereby $\Delta R$ 
$=\mathrm{R}_{0}-\mathrm{R}$ describes the distance of the outer shell with non-zero electron density. In this model the relation between lifetime $\tau$ and radii is given by

$$
\tau[\mathrm{ns}]=0.5 \times\left[1-\frac{\mathrm{R}}{\mathrm{R}_{0}}+\frac{\sin }{2 \pi}\right]^{-1}
$$

the empiric parameter $\Delta \mathrm{R}=\mathrm{R}_{0}-\mathrm{R}$ was shown to be $1.656 \AA$ [20].

Depth resolved determination of the pore size becomes possible by a pulsed positron beam with variable positron energy [22,23]. A positron flux of $10^{9} \mathrm{~s}^{-1}$ at a kinetic energy of $1 \mathrm{keV}$ provided by the positron source NEPOMUC at MLZ enables such a pulsed low-energy positron system (PLEPS) [21,22]. The mean implantation depth $\bar{z}$ for a given positron energy $\mathrm{E}$ can be approximated by the relationship

$$
\bar{z}=\left(\mathrm{A} / \mathrm{d}_{\mathrm{M}}\right) \mathrm{E}^{\mathrm{n}}
$$

where the material mass density $d_{M}$ and the material dependent parameters $A$ and $n$, which can be found in literature (e.g., [20]). For the present PALS calculations, we use the values of $\mathrm{A}=2.8 \mu \mathrm{g} \cdot \mathrm{cm}^{-2} \cdot \mathrm{keV}^{-\mathrm{n}}$ and $\mathrm{n}=1.7$, respectively.

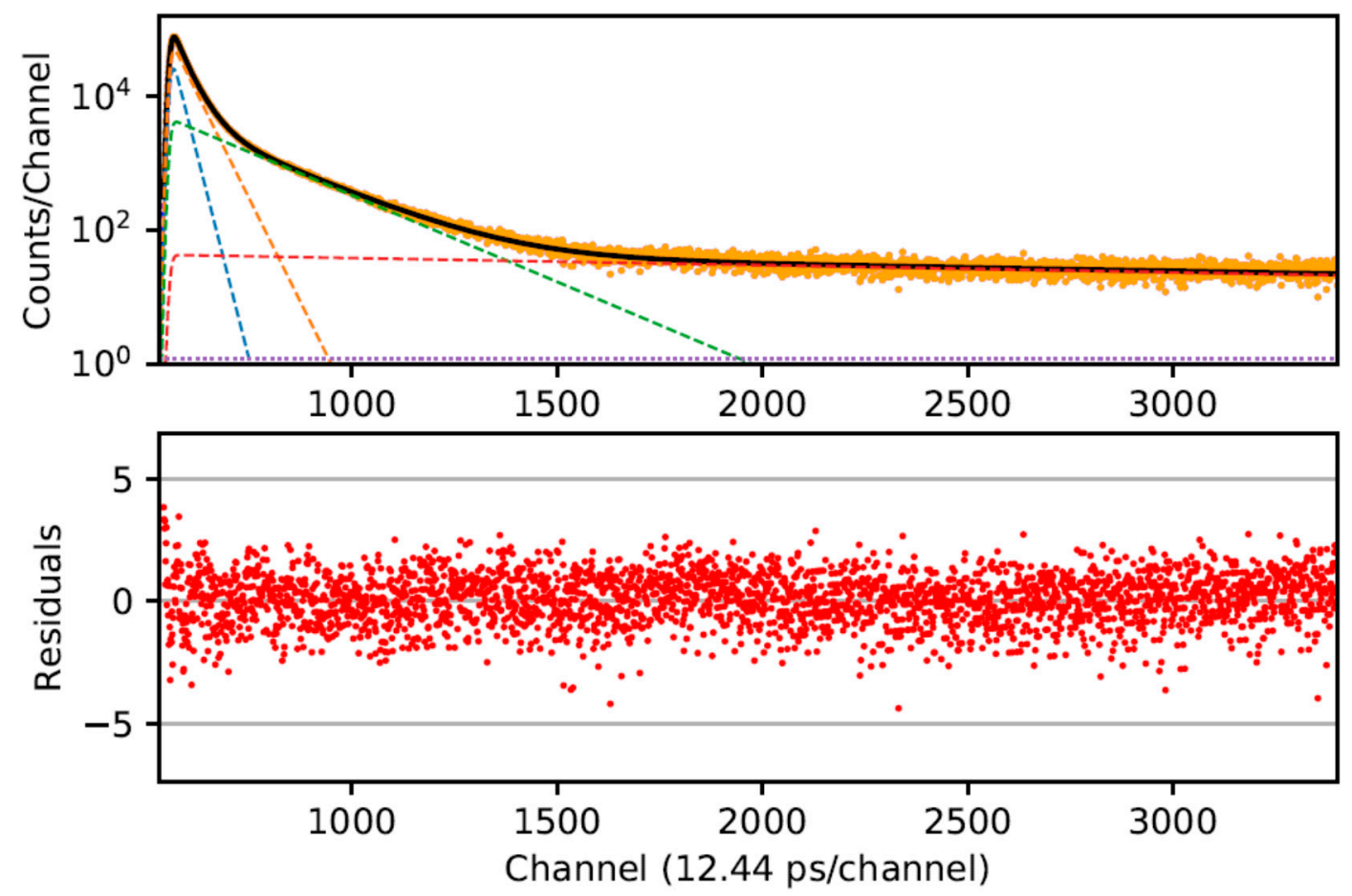

Figure A3. Typical PALS spectrum fitted with four lifetimes. The black line comprises four lifetime components (colored dashed lines) of a raw positron lifetime spectrum (dots) of the RO98pHt membrane recorded at a positron implantation energy of $8 \mathrm{keV}$.

Within polymeric systems, positrons annihilate according to at least three decay channels with different decay times $\tau_{i}(i=1,2,3, .$.$) . The lifetimes \tau_{1}$ and $\tau_{2}$ are related to direct positron annihilation (without positronium formation) whereby the shortest lifetime $\tau_{1}$ also comprises the annihilation of para-positronium (p-Ps). Both are in a 100 ps time range. The longer component $\tau_{3}$ is associated with the so-called pick-off lifetime of o-Ps in matter and extends to some ns. The time resolution of the instrument is in the range of 250 ps [22]. For data evaluation, the spectra are numerically deconvoluted by the measured resolution function and fitted with a least-squares method based on the Levenberg-Marquardt algorithm. For several spectra, reasonable fits are only obtained by taking into account an additional longer lifetime component with $\tau_{4}>\tau_{3}$. In this time regime the 
Tao-Eldrup model is not valid anymore, but pore sizes can be estimated using a classical description of the positronium-wall interaction [35]. Figure A3 shows a typical positron lifetime spectrum exhibiting four lifetimes $\tau_{i},(i=1-4)$. The decomposition into four components results in a reliable agreement between fit and recorded data as confirmed by the residuum (below). Note, that spectra recorded at PLEPS show a very low constant background of 1.3 counts per channel only. The intensity $\mathrm{I}_{3}$ allows us to observe changes in the free volume, as it is directly related to the total free volume of polymer melts. However, a reasonable value of free volume fraction on an absolute scale cannot be given, although $\mathrm{I}_{3}$ is considered to be proportional to the number of pores with a mean radius determined by $\tau_{3} . s$

\section{Abbreviations}

\begin{tabular}{|c|c|}
\hline SANS & small-angle neutron scattering \\
\hline PALS & positron-annihilation lifetime spectroscopy \\
\hline $\mathrm{d} \Sigma / \mathrm{d} \Omega(\mathrm{Q})$ & differential macroscopic cross-section \\
\hline $\mathrm{d} \Sigma / \mathrm{d} \Omega_{\text {inc }}$ & incoherent scattering cross-section. \\
\hline$\delta$ & scattering angle \\
\hline$\lambda$ & wavelength of neutron \\
\hline $\mathrm{k}$ & wavenumber of neutron $(2 \pi / \lambda)$ \\
\hline$\underline{\mathrm{Q}}$ & momentum transfer defined as $Q=4 \pi / \lambda \sin (\delta / 2)$ \\
\hline$\overline{\mathrm{Q}} 2$ & second moment with meaning of the invariant of scattering, \\
\hline$b_{i}$ & coherent scattering length of atom " $\mathrm{i}$ " \\
\hline$\Omega_{\mathrm{M}}$ & volume of molecule "M" \\
\hline$\rho_{\mathrm{M}}$ & coherent scattering length density of molecule "M" $\left(\rho_{M}=\Sigma b_{i} / \Omega_{M}\right)$ \\
\hline$\Phi_{\text {match }}$ & concentration of $\mathrm{D}_{2} \mathrm{O}$ in mixture of $\mathrm{H}_{2} \mathrm{O} / \mathrm{D}_{2} \mathrm{O}$ showing the same $\rho$ of the sample \\
\hline $\mathrm{R}_{\mathrm{g}}$ & radius of gyration \\
\hline $\mathrm{P}_{4}$ & Porod constant \\
\hline$\alpha$ & exponent of power law of $\mathrm{d} \Sigma / \mathrm{d} \Omega$ at $\mathrm{Q}>1 / \mathrm{R}_{\mathrm{g}}$ \\
\hline $\mathrm{V}_{\mathrm{p}}, \mathrm{S}_{\mathrm{p}}, \mathrm{N}_{\mathrm{p}}, \Phi_{\mathrm{p}}$ & volume, surface, number density, and volume fraction of domains exposed to scattering. \\
\hline $\mathrm{D}_{\mathrm{S}}$ & thickness of sample \\
\hline $\mathrm{RO}$ & reverse osmosis \\
\hline NF & nanofiltration \\
\hline TFC & thin film composite \\
\hline TMP & trans membrane pressure \\
\hline SSE & simulated secondary effluent \\
\hline SCF & supercritical fluid \\
\hline MA & methacrylic acid \\
\hline
\end{tabular}

\section{References}

1. Qasim, M.; Badrelzaman, M.; Darwish, N.N.; Darwish, N.A.; Hilal, N. Reverse osmosis desalination: A state-of-the-art review. Desalination 2019, 459, 59-104. [CrossRef]

2. Rodríguez-Calvo, A.; Silva-Castro, G.A.; Osorio, F.; González-López, J.; Calvo, C. Reverse osmosis seawater desalination: Current status of membrane systems. Desalin. Water Treat. 2015, 56, 849-861. [CrossRef]

3. Lee, K.P.; Arnot, T.C.; Mattia, D. A review of reverse osmosis membrane materials for desalination—Development to date and future potential. J. Membr. Sci. 2011, 370, 1-22. [CrossRef]

4. Pipich, V.; Schlenstedt, K.; Dickmann, M.; Kasher, R.; Meier-Haack, J.; Hugenschmidt, C.; Petry, W.; Oren, Y.; Schwahn, D. Morphology and porous structure of standalone aromatic polyamide films as used in RO membranes-An exploration with SANS, PALS, and SEM. J. Membr. Sci. 2019, 573, 167-176. [CrossRef]

5. Steiner, Z.; Rapaport, H.; Oren, Y.; Kasher, R. Effect of surface-exposed chemical groups on calcium-phosphate mineralization in water-treatment systems. Environ. Sci. Technol. 2010, 44, 7937-7943. [CrossRef]

6. Pipich, V.; Dahdal, Y.N.; Rapaport, H.; Kasher, R.; Oren, Y.; Schwahn, D. Effects of Biological Molecules on Calcium Mineral Formation Associated with Wastewater Desalination as Assessed using Small-Angle Neutron Scattering. Langmuir 2013, 29, 7607-7617. [CrossRef] 
7. Dahdal, Y.N.; Pipich, V.; Rapaport, H.; Oren, Y.; Kasher, R.; Schwahn, D. Small-Angle Neutron Scattering Studies of Mineralization on BSA coated Citrate capped Gold Nano-Particles used as a Model Surface for Membrane Scaling in RO Wastewater Desalination. Langmuir 2014, 30, 15072-15082. [CrossRef]

8. Schwahn, D.; Feilbach, H.; Starc, T.; Pipich, V.; Kasher, R.; Oren, Y. Design and Test of Reverse Osmosis Pressure Cell for in-situ Small-Angle Neutron Scattering Studies. Desalination 2017, 405, 40-50. [CrossRef]

9. Shtreimer, K.N.; Avisdris, T.; Arnusch, C.J.; Kasher, R. Grafted Polymer Coatings Enhance Fouling Inhibition by an Antimicrobial Peptide on Reverse Osmosis Membranes. Langmuir 2018, 35, 1935-1943. [CrossRef]

10. Werber, J.R.; Osuji, C.O.; Elimelech, M. Materials for next-generation desalination and water purification membranes. Nat. Rev. Mater. 2016, 1, 16018. [CrossRef]

11. Jiang, S.X.; Li, Y.N.; Ladewig, B.P. A review of reverse osmosis membrane fouling and control strategies. Sci. Total Environ. 2017, 595, 567-583. [CrossRef] [PubMed]

12. Heinz Maier-Leibnitz Zentrum. Available online: http://www.mlz-garching.de/instrumente-und-labore/ nanostrukturen/kws-3.html (accessed on 2 March 2020).

13. Alefeld, B.; Schwahn, D.; Springer, T. New Developments of Small-Angle Neutron Scattering Instruments with Focussing. Nucl. Instrum. Methods Phys. Res. 1989, A274, 210-216. [CrossRef]

14. Goerigk, G.; Varga, Z. Comprehensive Upgrade of the High-Resolution Small-Angle Neutron Scattering Instrument KWS-3 at FRM II. J. Appl. Crystallogr. 2011, 44, 337-342. [CrossRef]

15. Sears, V.F. Neutron scattering lengths and cross sections. Neutron News 1992, 3, 26-37. [CrossRef]

16. Gupta, V.B. Some developments of poly(ethylene terephthalate) fiber production and structure-property relationships. Indian J. Fibre Text. Res. 1995, 20, 43-59.

17. Fakirov, S.; Fischer, E.W.; Schmidt, G.F. Unit cell dimensions of poly (ethylene terephthalate. Makromol. Chem. 1975, 176, 2459-2465. [CrossRef]

18. Swift, G.; Bailey, J.; Kroschwitz, J.I. (Eds.) Acrylic (and methacrylic) acid polymers. In Encyclopedia of Polymer Science and Technology; Wiley: Hoboken, NJ, USA, 2003; Volume 1, pp. 79-96.

19. Jean, Y.C.; van Horn, J.D.; Hung, W.-S.; Lee, K.-R. Perspective of positron annihilation spectroscopy in polymers. Macromolecules 2013, 46, 7133-7145. [CrossRef]

20. Algers, J.; Sperr, P.; Egger, W.; Kögel, G.; Maurer, F. Median implantation depth implantation profile of 3-18 keV positrons in amorphous polymers. Phys. Rev. B 2003, 67, 12-14. [CrossRef]

21. Egger, W. Pulsed low energy positron system (PLEPS) at the Munich Research Reactor FRM II.pdf. Phys. Status Solidi 2007, 4, 3969-3972.

22. Sperr, P.; Egger, W.; Kögel, G.; Dollinger, G.; Hugenschmidt, C.; Repper, R.; Piochacz, C. Status of the pulsed low energy positron beam system (PLEPS) at the Munich Research Reactor FRM-II. Appl. Surf. Sci. 2008, 255, 35-38. [CrossRef]

23. Hugenschmidt, C.; Piochacz, C.; Reiner, M.; Schreckenbach, K. The NEPOMUC up-grade and advanced positron beam experiments. New J. Phys. 2012, 14, 055027. [CrossRef]

24. Lemmon, E.W.; McLinden, M.O.; Friend, D.G. Thermophysical Properties of Fluid Systems. In NIST Chemistry WebBook; Linstrom, P.J., Mallard, W.G., Eds.; NIST Standard Reference Database Number 69; National Institute of Standards and Technology: Gaithersburg, MD, USA; p. 20899. [CrossRef]

25. Kalebek, N.A.; Babaarslan, O. Fiber Selection for the Production of Nonwovens; IntechOpen: London, UK, 2016; Volume 1, pp. 1-32. [CrossRef]

26. Roe, R.J. Methods of X-ray and Neutron Scattering in Polymer Science; University Press: Oxford, UK, 2000.

27. de Gennes, P.-G. Scaling Concepts in Polymer Physics; Cornell University Press: Ithaca, NY, USA, 1979.

28. Dubrovski, P.D.; Brezocnik, M. Porosity and nonwoven fabric vertical wicking rate. Fibers Polym. 2016, 17, 801-808. [CrossRef]

29. Yoon, D.Y.; Flory, P.J. Small-angle neutron scattering by semicrystalline polyethylene. Polymer 1977, 18, 509-513. [CrossRef]

30. Ballard, D.G.H.; Cheshire, P.; Janke, E.; Nevin, N.; Schelten, J. Conformation of the molecules in drawn polypropylene revealed by neutron scattering. Polymer 1982, 23, 1875-1883. [CrossRef]

31. Hahn, K.; Kerth, J.; Zolk, R.; Schwahn, D.; Springer, T.; Kugler, J. Determination of the chain conformation in fast-spun polypropylene fibers by small-angle neutron scattering. Macromolecules 1988, 21, 1541-1543. [CrossRef] 
32. Song, X.; Gan, B.; Qi, S.; Guo, H.; Tang, C.Y.; Zhou, Y.; Gao, C. Intrinsic Nanoscale Structure of Thin Film Composite Polyamide Membranes: Connectivity, Defects, and Structure-Property Correlation. Environ. Sci. Technol. 2020. [CrossRef]

33. Eldrup, M.; Lightbody, D.; Sherwood, J.N. The temperature dependence of positron lifetimes in solid pxvalic acid. Chem. Phys. 1981, 63, 51-58. [CrossRef]

34. Olsen, J.V.; Kirkegaard, P.; Pedersen, N.J.; Eldrup, M. PALSfit: A new program for the evaluation of positron lifetime spectra. Phys. Status Solidi 2007, 4, 4004-4006. [CrossRef]

35. Gidley, D.W.; Peng, H.-G.; Vallery, R.S. Positron annihilation as a method to characterize porous materials. Annu. Rev. Mater. Res. 2006, 36, 49-79. [CrossRef]

36. Kłosowski, M.M.; McGilvery, C.M.; Li, Y.; Abellan, P.; Ramasse, Q.; Cabral, J.T.; Livingston, A.G.; Porter, A.E. Micro-to nano-scale characterisation of polyamide structures of the SW30HR RO membrane using advanced electron microscopy and stain tracers. J. Membr. Sci. 2016, 520, 465-476. [CrossRef]

37. Guinier, A. X-ray Diffraction; W.H. Freeman and Company: San Francisco, CA, USA, 1963.

38. Fujioka, T.; Oshima, N.; Suzuki, R.; Price, W.E.; Nghiem, L.D. Probing the internal structure of reverse osmosis membranes by positron annihilation spectroscopy: Gaining more insight into the transport of water and small solutes. J. Membr. Sci. 2015, 486, 106-118. [CrossRef]

39. Kim, S.H.; Kwak, S.Y.; Susuki, T. Positron annihilation spectroscopic evidence to demonstrate the flux-enhancement mechanism in morphology-controlled thin-film-composite (TFC) membrane. Environ. Sci. Technol. 2005, 39, 1764-1770. [CrossRef] [PubMed]

40. Fujioka, T.; Oshima, N.; Suzuki, R.; Khan, S.J.; Roux, A.; Poussade, Y.; Drewes, J.E.; Nghiem, L.D. Rejection of small and uncharged chemicals of emerging concern by reverse osmosis membranes: The role of free volume space within the active skin layer. Sep. Purif. Technol. 2013, 116, 426-432. [CrossRef]

41. Priestley, R.D.; Ellison, C.J.; Broadbelt, L.J.; Torkelson, J.M. Structuaral relaxation of polymer glasses at surfaces, interfaces, and in between. Science 2005, 309, 456-459. [CrossRef]

42. Freger, V. Nanoscale Heterogeneity of Polyamide Membranes Formed by Interfacial Polymerization. Langmuir 2003, 19, 4791-4797. [CrossRef]

43. Beaucage, G. Approximations leading to a unified exponential power-law approach to small-angle scattering. J. Appl. Crystallogr. 1995, 28, 717-728. [CrossRef]

44. Frielinghaus, H. Strategies for removing multiple scattering effects revisited. Nucl. Instrum. Methods Phys. A 2018, 904, 9-14. [CrossRef]

45. Schelten, J.; Schmatz, W. Multiple Scattering Treatment for Small-Angle Scattering Problems. J. Appl. Cryst. 1980, 13, 385-390. [CrossRef]

46. Vestergaard Jensen, G.; Barker, J.G. Effects of multiple scattering encountered for various small-angle scattering model functions. J. Appl. Cryst. 2018, 51, 1455-1466. [CrossRef]

47. Program. Available online: https://jugit.fz-juelich.de/sans/muscatt (accessed on 2 March 2020).

48. Pipich, V.; Schwahn, D. Densification of supercritical carbon cioxide (CO2) accompanied by droplet formation when passing the Widom line. Phys. Rev. Lett. 2018, 120, 145701. [CrossRef]

49. Hugenschmidt, C.; Ceeh, H. The free volume in dried and H2O-loaded biopolymers studied by positron lifetime measurements. J. Phys. Chem. B 2014, 118, 9356-9360. [CrossRef] [PubMed]

(C) 2020 by the authors. Licensee MDPI, Basel, Switzerland. This article is an open access article distributed under the terms and conditions of the Creative Commons Attribution (CC BY) license (http://creativecommons.org/licenses/by/4.0/). 